\title{
Clearing spheroids for 3D fluorescent microscopy: combining safe and soft chemicals with deep convolutional neural network
}

\author{
Ali Ahmad ${ }^{1,2}$, Saba Goodarzi ${ }^{3}$, Carole Frindel ${ }^{2}$, Gaëlle Recher ${ }^{4}$, Charlotte Riviere ${ }^{3,5}$, \\ David Rousseau ${ }^{1, *}$, \\ 1 Laboratoire Angevin de Recherche en Ingénierie des Systèmes (LARIS), UMR INRAE \\ IRHS, Université d'Angers, Angers, 49000, France \\ 2 Centre de Recherche en Acquisition et Traitement de l'Image pour la Santé \\ (CREATIS) , CNRS UMR 5220 - INSERM U1206, Université Lyon 1, Insa de Lyon, \\ Villeurbanne, 69100, France \\ 3 Univ Lyon, Université Claude Bernard Lyon 1, CNRS UMR-5306, Institut Lumière \\ Matière, F-69622, Villeurbanne, 69100, France \\ 4 BioImaging \& OptoFluidics, LP2N, CNRS UMR5298, IOGS, Université de Bordeaux, \\ Talence, F-33400, France \\ 5 Institut Universitaire de France (IUF), France \\ * david.rousseau@univ-angers.fr
}

\begin{abstract}
In life sciences, there are increasing interest in 3D culture models to better reproduce the $3 \mathrm{D}$ environment encountered in-vivo. Imaging of such 3D culture models is instrumental for drug discovery, but face several issues before its use becomes widespread. Extensive microscopic investigation of these 3D cell models faces the challenge of light penetration in depth in opaque biological tissues. To overcome this limit, diverse clearing techniques have emerged over the past decades. However, it is not straightforward to choose the best clearing protocols, and assess quantitatively their clearing efficiency. Focusing on spheroids, we propose a combination of fast and cost-effective clearing procedure for such medium-sized samples. A generic method with local contrast metrics and deep convolutional neural network-based segmentation of nuclei is proposed to quantify the efficiency of clearing. We challenged this method by testing the possibility to transfer segmentation knowledge from a clearing protocol to another. The later results support the pertinence of training deep learning algorithms on cleared samples to further use the segmentation pipeline on non-cleared ones. This second step of the protocol gives access to digital clearing possibilities applicable to live and high-throughput optical imaging.
\end{abstract}

\section{Introduction}

Spheroids are three-dimensional (3D), heterogeneous aggregates of proliferating, quiescent and necrotic in vitro cell culture systems 11. They have gained increasing interest in drug screening because of their ability to closely mimic the main features of physiological cell to cell and cell to matrix contacts as in in vivo human solid tumors 2, 3]. Because of the standardization of their production, spheroids became a model of choice in the context of the $3 \mathrm{R}$ (replace, reduce, refine) notably for 
high-throughput drug screening 4,5. Yet, the experimental readouts are often quantitative, between each size-controlled spheroid, but fail at providing insights in regards with single-cell information within the spheroid itself, due to its lack of amenability for in-depth microscopy. One main challenge is the visualization of spheroids via fluorescence imaging due to the light scattering inducing limited depth penetration in such opaque structure and therefore low data analysis performance at a single-cell level 6.7]. One solution to enhance visualization of fluorescently-labelled spheroids is to use optical clearing techniques, which improve in-depth imaging and allow high quality image acquisition and high-throughput image analysis. The rough principle of optical clearing is to uniformise the optical index within the 3D structure, so as to minimise diffraction. One of the option is to remove the lipids that contribute to light scattering effects, or, to replace water by a solution closer to the lipid refractive index 8 13. So far, assessment of the quality of clearing in 3D spheroids has been performed with local quality metrics such as signal to noise ratio (SNR) and contrast to noise ratio (CNR) 14 16. However, it is not straight-forward to relate local metrics with final quantitative measures of interest. We not only propose to revisit the assessment of clearing quality in such a perspective, but we also provide an assessment based on segmentation metrics.

Final quantitative measures for high-throughput image analysis of spheroids mostly rely on whole-spheroid fluorescent measures, size analysis and matrix invasion as an indicator of drug anti metastatic effects 17,22 . These metrics provide useful macroscopic quantitative information but do not provide enough biological details at single cell level for assessing therapy responses nor quantitative analysis such as cell counting and aggregation studies, and also no characteristics over time such as growth ratio and proliferation ratio. To overcome these limitations, individual cell nuclei segmentation methods have been developed for 2D and 3D spheroid images 23 30. State-of-the-art methods for cell nuclei segmentation in microscopy are currently the deep learning-based methods. Also, a specific interest of the deep learning-based approach, by contrast with a standard image processing pipeline composed of denoising step [31] followed by a segmentation step [32 33], is to offer an end-to-end learning process where all the pipeline is optimized at the same time. Several architectures were developed to segment nuclei by integrating two or more channels in the output of deep learning architecture or by applying post processing methods to the predicted segmentation maps to enhance segmentation quality 34 40. Deep learning methods were applied to segment entire spheroids of different sizes, shapes, and illumination conditions [41] and also to segment nuclei of 3D spheroid images [42]. In these closely related work, only one clearing method was investigated. By contrast in this article we propose, with a deep learning perspective, a protocol to compare clearing methods for the segmentation of nuclei in spheroids under 3D fluorescence microscopy.

A scheme of the operating pipeline of the article is provided in Figure 1 . We compare clearing protocols RapiClear and Glycerol (Figure11.A) to non cleared samples (Control). The two clearing methods investigated have been chosen for their simplicity and non-toxicity. Both Rapiclear 43] and Glycerol [15] have already been reported as fast and cost-effective yet efficient clearing procedures, for medium-sized samples such as organoids and spheroids of few hundreds of micrometers. First, we assess the clearing quality of spheroid images acquired with confocal fluorescence microscopy with the conventional local metrics such as signal to noise ratio (SNR) and contrast to noise ratio (CNR) (Figure 1.B). Then, we use deep learning segmentation methods to assess the quality of the clearing (Figure 1.C,D). Finally, we investigate the possibility of digital clearing of non-cleared data with cleared data models via transfer learning and the transferability of knowledge from a clearing protocol to another (Figure 1.E). In this manuscript, we show that using a simple deep learning strategy, it is possible to get 
reliable segmented images even for images with low intensity and low signal-to-noise ratio. Also, we demonstrate the segmentation knowledge transferability from cleared samples to native tissues for fast digital-clearing of living specimens on the fly.

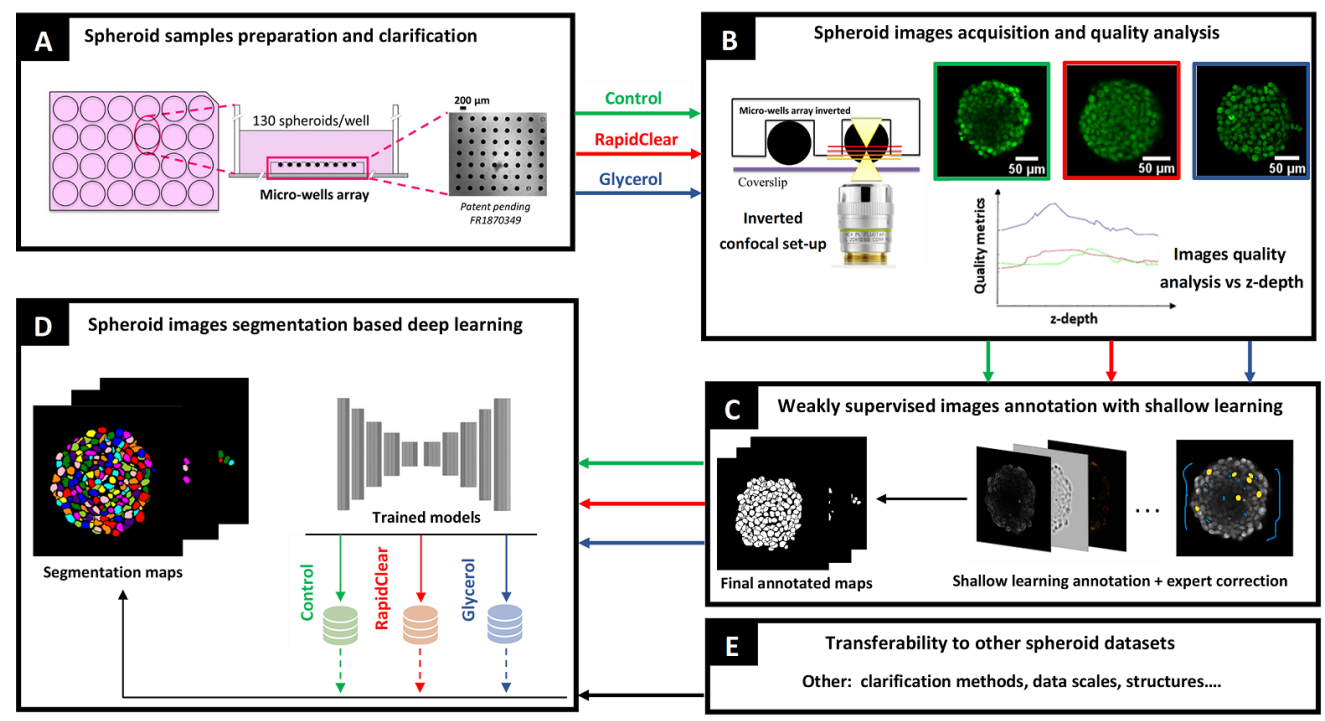

Fig 1. Protocol pipeline: (A) Spheroid samples are cultured in micro-well arrays. Control are the non cleared samples and others are clarified with two distinct clearing methods Rapiclear and Glycerol. (B) Then, spheroid images acquired with a confocal microscope are analyzed for quality assessment along z-depth. (C) A dataset of images for each clarification method is annotated using shallow learning and corrected by an expert. (D) Finally, deep learning models are trained with the annotated data sets and the three segmentation models trained on Rapiclear, Control and Glycerol are tested for (E) data transferability on various clarification methods.

\section{Results and Discussion}

\section{Comparison of clearing methods with local metrics}

We assessed the efficiency of RapiClear and Glycerol clearing protocols on image quality by comparing their datasets with non cleared datasets (Control) using image local quality metrics evaluation. This was quantitatively evaluated in depth on the 3D spheroid stacks (z-depth) based on the computation of various local metrics using patches cropped from the center of the spheroid signal in each slice (see SI Figures 1.A) The used metrics were the signal to noise ratio (SNR) (Equation 1) and the contrast to noise ratio (CNR) calculated by two ways: from Bhattacharyya coefficient (BC) (Equation 2) and from Fisher ratio (FR) (Equation 3). Figure 2 shows the xz planes at the center of spheroids and the xy planes at selected depth (from $70 \%$ to $100 \%$ of maximum diameter, corresponding to the range of depth where the three conditions can be compared) also the mean and the standard deviation (std) of the normalized average intensity (average I/Imax) evolution and of the computed local metrics from three spheroids for each Control, RapiClear and Glycerol conditions. Visual qualitative inspection of the $\mathrm{xz}$ and $\mathrm{xy}$ planes of spheroids are provided in Fig 2,A,B. Quantitative evaluation of image quality (Fig 2 C) shows the degradation of image with depth $\mathrm{z}$. 
bioRxiv preprint doi: https://doi.org/10.1101/2021.01.31.428996; this version posted February 1, 2021. The copyright holder for this preprint (which was not certified by peer review) is the author/funder. All rights reserved. No reuse allowed without permission.

Concerning intensities, an important drop is recorded for all clearing methods. This drop is more pronounced in the non cleared (control) spheroids. The intensity evolutions with depth are similar for Rapiclear and Glycerol. SNR and FR metrics appear almost constant for the three investigated clearing conditions while BC shows global degradation along depth z. For the three metrics SNR, FR and BC, Glycerol shows significantly better values by comparison with Rapiclear and Control. However, it is uneasy to produce a secured prediction of the effect of clearing along $\mathrm{z}$ with local metrics. This is why it was important also to confront what would be found with conventional local metrics with a machine learning perspective.

A.
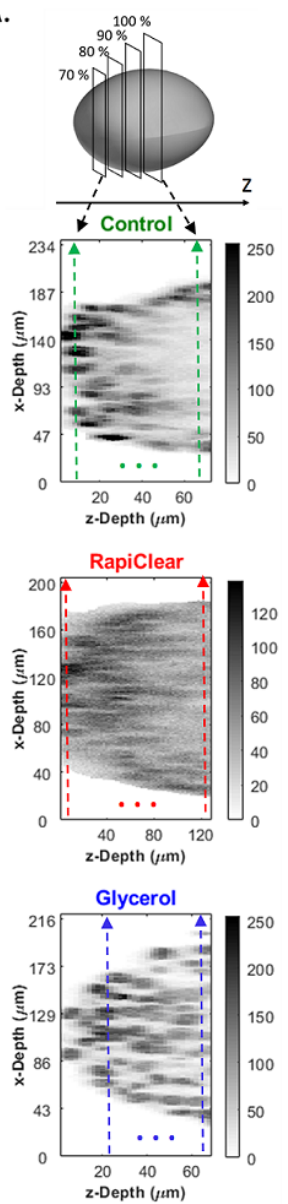

\section{B.}

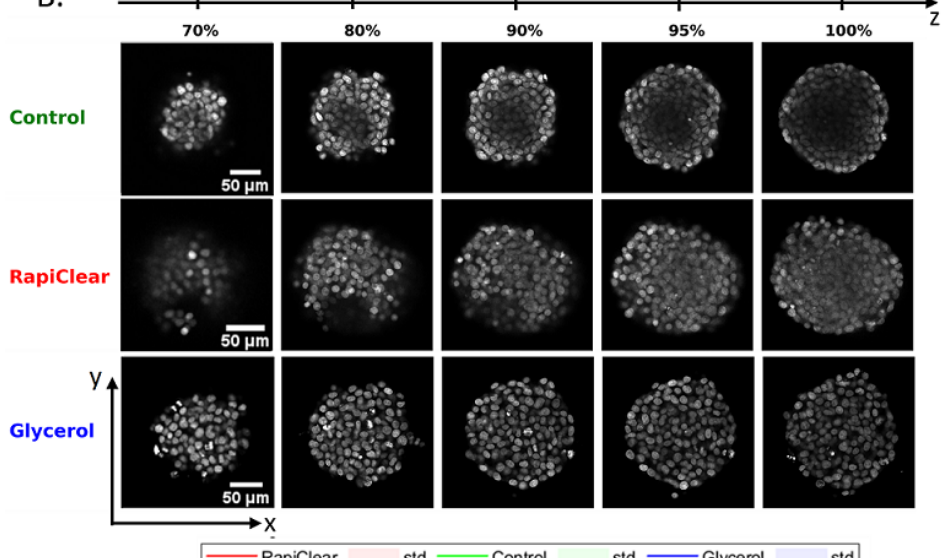

C.
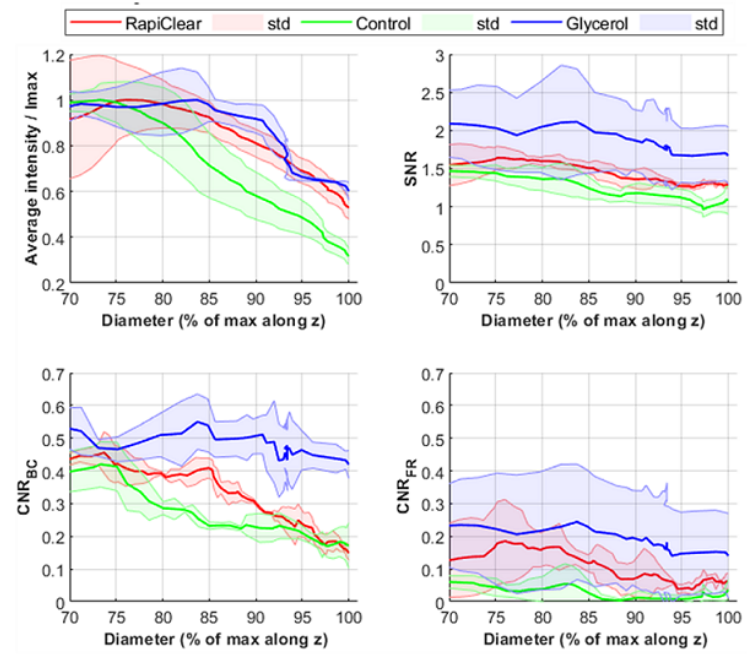

Fig 2. Image quality analysis function of the depth $(z)$ defined as the percentage of the maximum diameter for the datasets produced with Control, RapiClear and Glycerol clarification methods. Slices with $100 \%$ of diameter are considered as the center of the $3 \mathrm{D}$ spheroids. (A) Illustration of the $x z$ slices. Colored dashed lines correspond to the location (in $\mu m$ ) of the slices $x y$ with $70 \%$ and $100 \%$ of maximum diameter. (B) Visualization of test images (xy slices) normalized in depths $(z)$ and used to evaluate segmentation performance for each clarification method. (C) The mean and standard deviation (std) of the average intensity, SNR, $C N R_{B C}$ by Battacharayya and $C N R_{F R}$ by Fisher ratio computed for 3 spheroids for each clarification method and plotted as a function of the percentage of maximum diameter. 


\section{Spheroid 2D images segmentation}

We used deep learning segmentation methods to characterize the quality of spheroid clearing protocols. Several segmentation methods such as Unet, Dist and Stardist, associated with post-processing steps based on dynamic morphology reconstruction and watershed algorithm (DM) were applied to the test images of Figure 2.B produced from the tested clearing conditions. We used the F1-score to quantify the quality of segmentation as a pixel wise metric and the aggregated Jaccard Index (AJI) as an object wise metric. We tested the segmentation efficiency for two cases: (i) when test images and pre-trained segmentation models are from the same clearing method and (ii) in a cross way taking test images from a clearing condition and using pre-trained model from another. Table 1 shows the mean values and the standard deviations (std) of the F1-score and AJI computed for test images located at 70, 80, 90,95 and $100 \%$ of maximum diameter along $\mathrm{z}$.

Interestingly, in accordance with the observation recorded by the local quality metrics, Glycerol clearing protocol provides the best segmentation performance in all tested configurations (Table 1.C-3). In addition, it is remarkable that Glycerol segmentation model is also powerful to segment data sets from Control or from Rapiclear clearing when segmentation method is properly chosen (Table 1 A-3,B-3). It is also striking to note that segmentation results of Control and RapiClear test images gives higher F1-score and AJI values with segmentation models trained on Glycerol than with models trained on their data. As shown in Fig. 3.A, these average results are almost stationary along $\mathrm{z}$ for all clearing methods tested (also see qualitative illustration of the final segmentation in Figure 3 . B and SI Figures 2 and 3.A. This demonstrates that the local evolution in intensity or SNR shown in Figure 2 2 do not necessarily correlate with the segmentation performances.

\section{Segmentation models transferability to other data sets}

Finally, we tested the transferability from cleared datasets to another datasets by segmenting test images from another clearing methods, another structure and also another imaging system using pre-trained segmentation models of Control, Rapiclear and Glycerol. For this study, we used colorectal carcinoma HCT-116 cell lines test images cleared with TDE and acquired with a laser scan confocal system (Nikon). Also, we selected two datasets from the literature such as Breast carcinoma T47D cells cleared with ScaleS method and acquired with a spinnig disk confocal system (Opera Phenix) 23. and colorectal carcinoma HCT-116 cell line cleared with CUBIC method and acquired with a light-sheet fluorescence (LSF) microscope 44]. Table 1.D,E,F shows the mean and standard deviation of computed F1-score and AJI segmentation metrics for each test images produced from TDE, ScaleS and CUBIC clearing methods and segmented with the Control, RapiClear and Glycerol pre-trained models. The segmentation results are high for both TDE and ScaleS (see Table 1 and Figure 4.A). Similarly to what was found for Rapiclear and Control, Glycerol appears, for both TDE and ScaleS also, as the best method to train on to benefit from transfer learning. This experiment clearly proves the feasibility of information transferability by digital clearing from clearing method to another acquired with the same imaging system but with different structure and data scale. Contrariwise, CUBIC dataset acquired with light-sheet fluorescence (LSF) microscopy and presenting very different contrast and artefacts shows low performance. This experiment points the limit of data transferability by digital clearing (see Figure 4.B and SI Figures 3.B and 4 for qualitative illustration of final segmentation maps). Figure 5 A illustrates the qualitative interest of digital clearing available at the output of neural networks when the best segmentation neural network (Dist segmentation) is trained on the best 
bioRxiv preprint doi: https://doi.org/10.1101/2021.01.31.428996; this version posted February 1, 2021. The copyright holder for this preprint (which was not certified by peer review) is the author/funder. All rights reserved. No reuse allowed without permission.

\begin{tabular}{|c|c|c|c|c|c|c|c|c|c|}
\hline \multirow{2}{*}{ Test data } & \multirow{2}{*}{ Segmentation models } & \multicolumn{3}{|c|}{ F1-score \pm std } & \multicolumn{5}{|c|}{$\mathbf{A J I} \pm \mathbf{s t d}$} \\
\hline & & Unet & Dist & Stardist & Unet & Unet+DM & Dist & Dist $+D M$ & Stardist \\
\hline \multirow{6}{*}{$\begin{array}{l}\text { Control } \\
\text { (A) }\end{array}$} & Control & 0.85 & 0.85 & 0.84 & 0.07 & 0.51 & 0.26 & 0.52 & 0.59 \\
\hline & (1) & \pm 0.01 & \pm 0.01 & \pm 0.01 & \pm 0.06 & \pm 0.03 & \pm 0.05 & \pm 0.03 & \pm 0.03 \\
\hline & RapiClear & 0.84 & 0.85 & 0.72 & 0.14 & 0.52 & 0.16 & 0.52 & 0.42 \\
\hline & (2) & \pm 0.02 & \pm 0.01 & \pm 0.04 & \pm 0.03 & \pm 0.05 & \pm 0.03 & \pm 0.03 & \pm 0.07 \\
\hline & Glycerol & 0.86 & 0.86 & 0.84 & 0.01 & 0.57 & 0.06 & 0.55 & 0.55 \\
\hline & (3) & \pm 0.02 & \pm 0.01 & \pm 0.02 & \pm 0.01 & \pm 0.05 & \pm 0.04 & \pm 0.02 & \pm 0.06 \\
\hline \multirow{6}{*}{$\begin{array}{l}\text { RapiClear } \\
\text { (B) }\end{array}$} & Control & $\overline{0.82}$ & 0.83 & 0.81 & 0.03 & 0.41 & 0.07 & 0.47 & 0.52 \\
\hline & (1) & \pm 0.01 & \pm 0.01 & \pm 0.01 & \pm 0.02 & \pm 0.02 & \pm 0.03 & \pm 0.05 & \pm 0.03 \\
\hline & RapiClear & 0.83 & 0.84 & 0.76 & 0.11 & 0.46 & 0.08 & 0.48 & 0.43 \\
\hline & (2) & \pm 0.01 & \pm 0.01 & \pm 0.03 & \pm 0.09 & \pm 0.04 & \pm 0.04 & \pm 0.02 & \pm 0.06 \\
\hline & Glycerol & 0.83 & 0.83 & 0.80 & 0.01 & 0.46 & 0.03 & 0.47 & 0.49 \\
\hline & (3) & \pm 0.03 & \pm 0.02 & \pm 0.03 & \pm 0.01 & \pm 0.02 & \pm 0.01 & \pm 0.03 & $\pm \mathbf{0 . 0 3}$ \\
\hline \multirow{6}{*}{$\begin{array}{l}\text { Glycerol } \\
\text { (C) }\end{array}$} & Control & $\overline{0.89}$ & $\overline{c 0.89}$ & $\overline{0.89}$ & $\overline{0.29}$ & $\overline{0.63}$ & $\overline{0.46}$ & 0.64 & $\overline{0.68}$ \\
\hline & (1) & \pm 0.01 & \pm 0.01 & \pm 0.01 & \pm 0.01 & \pm 0.01 & \pm 0.06 & \pm 0.03 & \pm 0.02 \\
\hline & RapiClear & 0.88 & 0.87 & 0.78 & 0.40 & 0.63 & 0.46 & 0.62 & 0.51 \\
\hline & (2) & \pm 0.01 & \pm 0.01 & \pm 0.02 & \pm 0.07 & \pm 0.03 & \pm 0.04 & \pm 0.02 & \pm 0.03 \\
\hline & Glycerol & 0.91 & 0.91 & 0.90 & 0.34 & 0.70 & 0.47 & 0.71 & 0.70 \\
\hline & (3) & \pm 0.01 & \pm 0.01 & \pm 0.01 & \pm 0.07 & \pm 0.02 & \pm 0.05 & \pm 0.02 & \pm 0.02 \\
\hline \multirow{6}{*}{$\begin{array}{l}\text { TDE } \\
\text { (D) }\end{array}$} & Control & 0.88 & 0.88 & 0.84 & 0.02 & 0.44 & 0.06 & 0.56 & 0.59 \\
\hline & (1) & \pm 0.01 & \pm 0.02 & \pm 0.02 & \pm 0.01 & \pm 0.07 & \pm 0.05 & \pm 0.05 & \pm 0.04 \\
\hline & RapiClear & 0.89 & 0.89 & 0.78 & 0.08 & 0.60 & 0.09 & 0.60 & 0.52 \\
\hline & (2) & \pm 0.01 & \pm 0.02 & \pm 0.02 & \pm 0.04 & \pm 0.01 & \pm 0.04 & \pm 0.02 & \pm 0.06 \\
\hline & Glycerol & 0.90 & 0.89 & 0.84 & 0.02 & 0.39 & 0.02 & 0.54 & 0.58 \\
\hline & (3) & $\pm \mathbf{0 . 0 2}$ & \pm 0.03 & \pm 0.02 & \pm 0.02 & \pm 0.05 & \pm 0.02 & \pm 0.02 & $\pm \mathbf{0 . 0 4}$ \\
\hline \multirow{6}{*}{$\begin{array}{l}\text { ScaleS } \\
\text { (E) }\end{array}$} & Control & 0.85 & 0.86 & 0.83 & 0.29 & 0.54 & 0.39 & 0.59 & 0.62 \\
\hline & (1) & \pm 0.03 & \pm 0.03 & \pm 0.03 & \pm 0.13 & \pm 0.03 & \pm 0.1 & \pm 0.05 & \pm 0.05 \\
\hline & RapiClear & 0.83 & 0.85 & 0.77 & 0.22 & 0.58 & 0.26 & 0.58 & 0.50 \\
\hline & (2) & \pm 0.06 & \pm 0.05 & \pm 0.06 & \pm 0.1 & \pm 0.03 & \pm 0.13 & \pm 0.06 & \pm 0.06 \\
\hline & Glycerol & 0.87 & 0.85 & 0.85 & 0.12 & 0.57 & 0.14 & 0.56 & 0.63 \\
\hline & (3) & \pm 0.03 & \pm 0.03 & \pm 0.03 & \pm 0.07 & $\pm \mathbf{0 . 0 4}$ & \pm 0.11 & \pm 0.05 & \pm 0.03 \\
\hline \multirow{6}{*}{$\begin{array}{c}\text { CUBIC } \\
(\mathrm{F})\end{array}$} & Control & 0.68 & 0.69 & 0.24 & 0.003 & 0.33 & 0.004 & 0.16 & 0.07 \\
\hline & (1) & \pm 0.002 & \pm 0.03 & \pm 0.04 & \pm 0.001 & \pm 0.04 & \pm 0.001 & \pm 0.04 & \pm 0.02 \\
\hline & RapiClear & 0.66 & 0.63 & 0.42 & 0.003 & 0.23 & 0.003 & 0.19 & 0.11 \\
\hline & $(2)$ & \pm 0.01 & \pm 0.02 & \pm 0.02 & \pm 0.001 & \pm 0.03 & \pm 0.001 & \pm 0.02 & \pm 0.001 \\
\hline & Glycerol & 0.58 & 0.58 & 0.26 & 0.002 & 0.23 & 0.002 & 0.16 & 0.06 \\
\hline & (3) & \pm 0.01 & \pm 0.01 & \pm 0.04 & \pm 0.001 & \pm 0.04 & \pm 0.001 & \pm 0.01 & \pm 0.01 \\
\hline
\end{tabular}

Table 1. Mean and standard deviation of the computed segmentation metric (F1-score, AJI) for five test images produced from Control, RapiClear and Glycerol. Tests are realized in cross way between the segmentation models (A,B and C). And also for five test images produced from TDE, ScaleS and CUBIC to test data transferability from a clearing protocol to another clearing protocol (D, E and F). Various segmentation methods are used. The bold values are best segmentation model for each clearing method datasets. Except for Cubic clearing method where all methods provide low performance, Glycerol appears as the best method on which one should train the deep learning models in order to benefit from digital clearing.

clearing method (Glycerol) and applied to uncleared data or cleared with Rapiclear. It is obvious with Fig. 5. A that the intensity attenuation recorded with local metrics along z can be compensated with the deep neural network thanks to digital clearing. As shown in Fig. 5 . B in comparison with ground truth, a significant improvement of the counting of cells along $\mathrm{z}$ is brought by training on images obtained with the best clearing method while applying the model directly on control or on a less efficient clearing method. This opens also the possibility of an efficient post-segmentation 3D reconstruction of full spheroids (see SI Figure 5 and SI videos). 
A.
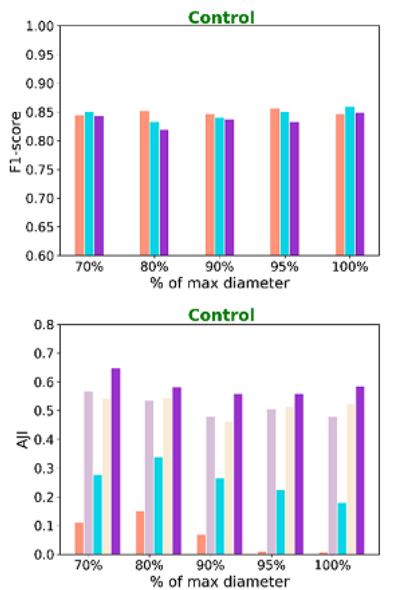

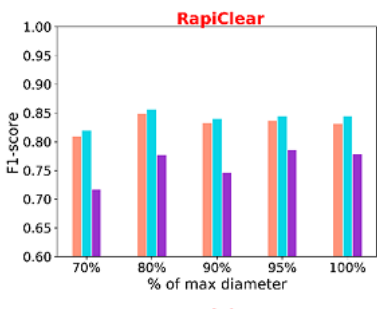

Rapiclear

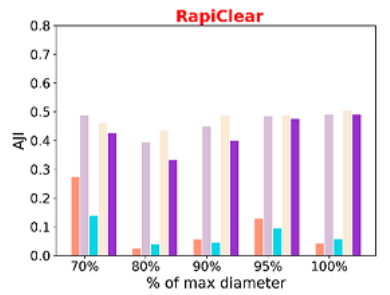

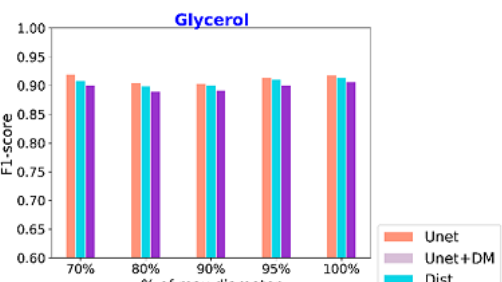

Dist

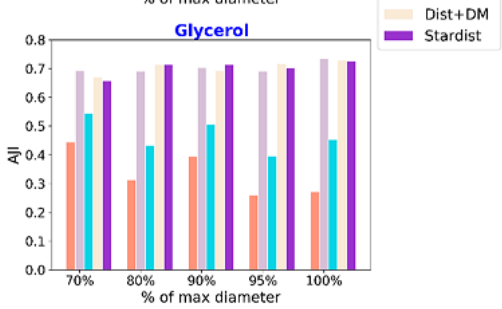

B.

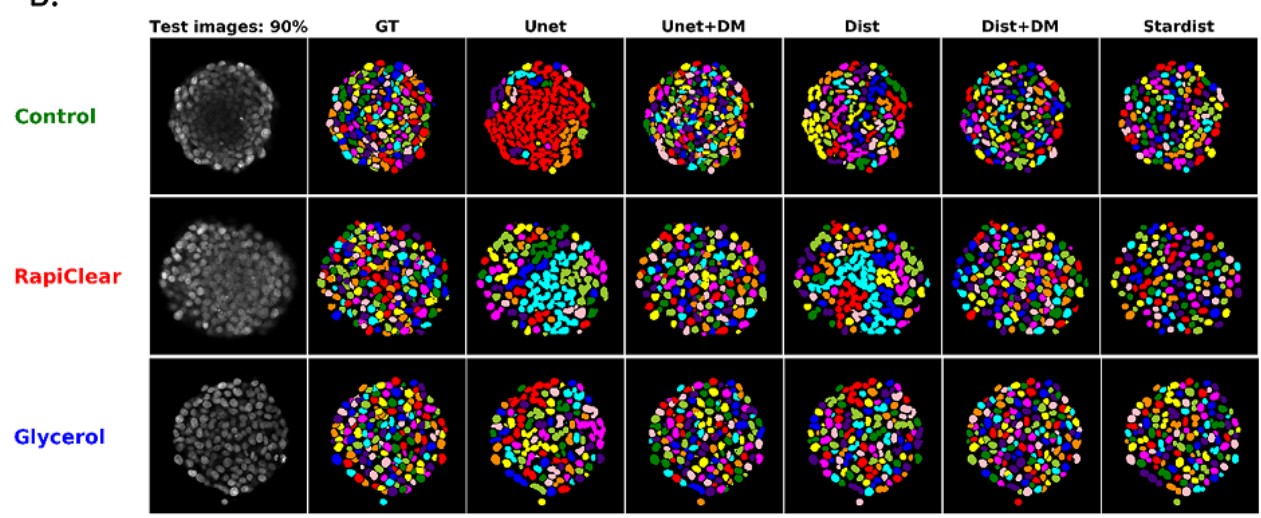

Fig 3. Segmentation results as a function of depths (z). (A) Quantitative segmentation results (F1-score, AJI) for the five test images taken at depth ranging from 70, 80,90, 95 and 100\% defined as the percentage of maximum diameter. Segmentation methods are trained and tested on the same clearing method. (B) Qualitative illustration of the final segmentation of the slices at $90 \%$ of maximum diameter produced from Control, RapiClear and Glycerol clearing methods.

\section{Conclusion}

In this report, we have investigated the interest of a deep learning perspective for the comparison of clearing methods in fluorescence microscopy. This was illustrated for a task of nuclei segmentation in spheroids under 3D fluorescent microscopy. The best clearing method identified with conventional local metric follows the performance of state-of-the-art deep learning based segmentation methods. However, because the training datasets include images from various depth, no real influence of depth was observed on segmentation performance contrarily to what was found with local metric. This demonstrates that local metric can be used to select an optimal clearing method but that final performances can be made invariant to the remaining noise thanks to adequate machine learning strategies.

We specially tested the interest of transfer learning in this context. We investigated the interest to train on one clearing condition and to test on another. The best 
A.
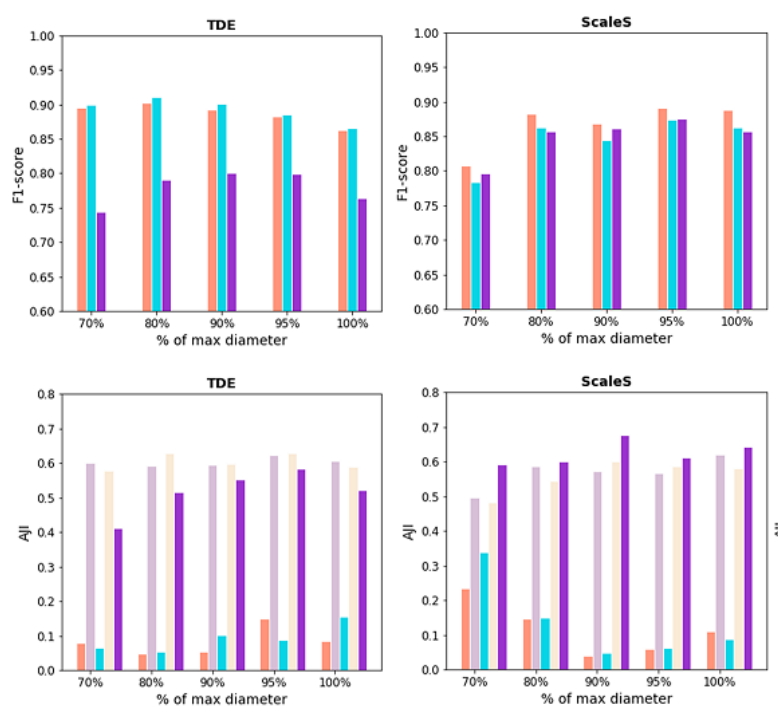

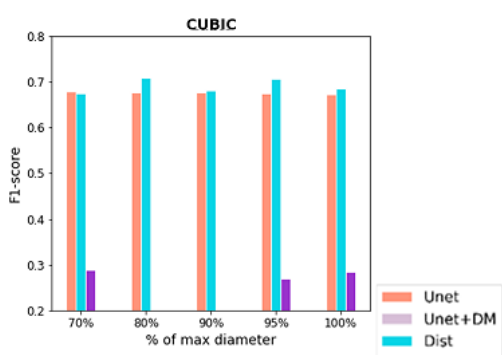

Dist+DM

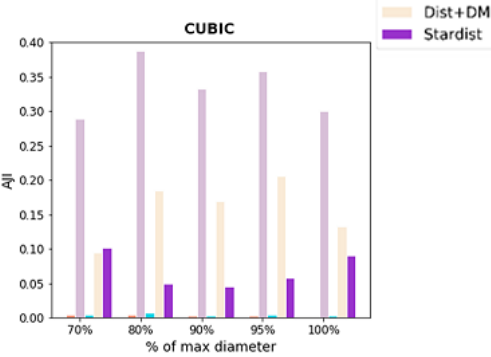

B.

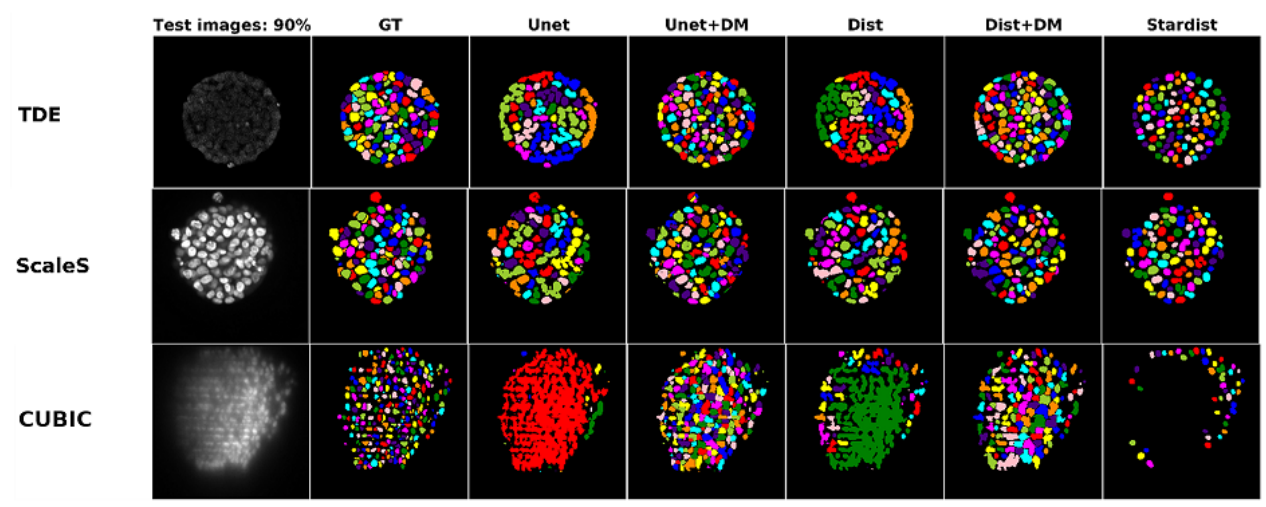

Fig 4. Data transferability assessment: segmentation results as a function of depths $(z)$ defined as the percentage of maximum diameter. (A) Quantitative segmentation results (F1-score, AJI) for the five test images produced from TDE, ScaleS and CUBIC clearing methods and segmented using the RapiClear, Glycerol and Control segmentation models respectively. (B) Qualitative illustration of the final segmentation results for the slices at $90 \%$ of maximum diameter.

segmentation method was also found to be the one which gave the best transfer results. This is specially interesting when the test images are produced in uncleared conditions. Training a model on the best cleared conditions enable to perform segmentation of nuclei in non cleared conditions with enhanced performances. This opens the way to digital clearing of the samples. Annotated cleared images of high quality produced with time consuming protocols could enable anyone to denoise images acquired with much simpler protocols. For this reason we release our annotated data set publicly as pointed in section S1 Dataset

These results could be extended in various directions. The inference of the proposed segmentation approach is very fast (around 2 minutes for a stack of 41 slices of $40 \mathrm{Mbs}$ with a processor Intel Core i7-6700HQ CPU @ $2.60 \mathrm{GHz}$ ). It can be easily used for on the fly segmentation during acquisition for fixed samples, as well as for live imaging (for

\author{
160
} 161 162 
A.

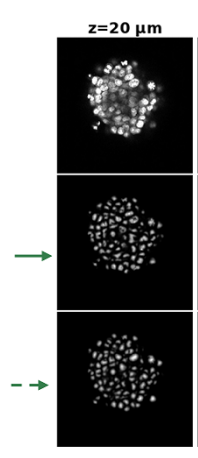

Control

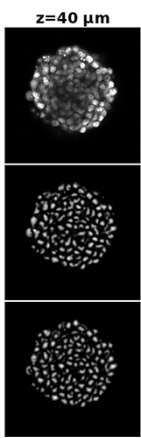

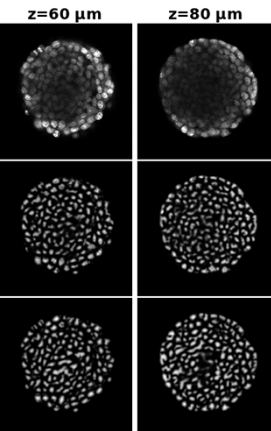

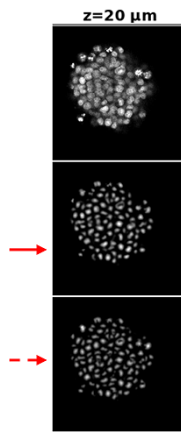

$\begin{array}{lll}\text { - Control } & --- & \text { Control with Digital Clearing } \\ \text { - Rapiclear } & --- & \text { RapiClear with Digital Clearing }\end{array}$
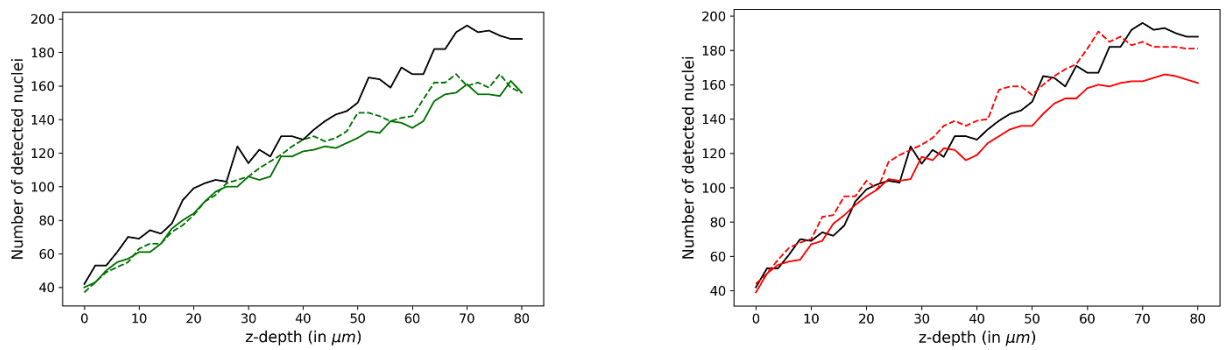

Fig 5. Digital clearing effect analysis. (A) Slices of non cleared spheroid (control) and same spheroid cleared with RapiClear are illustrated function of z-depth $(\mu m)$. The predicted distance maps at the output of Unet architecture for Dist segmentation method computed for the slices with control model (green solid line) and RapiClear model (red solid line) and also with digital clearing via glycerol model (green and red dashed lines). (B) Number of detected nuclei computed after Dist segmentation function of the depth $\mathrm{z}$ in $\mu \mathrm{m}$ for the same non cleared and cleared spheroid before and after digital clearing. Ground truth in black solid line corresponds to a manual counting by an expert.

experiments where samples are acquired over extended time-period [tens of hours], but with rather long time intervals [higher then $10 \mathrm{~min}]$ ). By bringing such single cell metrics directly available to the user, it opens up promising applications to screen therapeutics drugs within 3D environment closer to in-vivo. Colorectal cancer spheroids were used here as an example. The method is also readily available for organoïds that have emerged as very powerful in vitro models to mimic various normal and pathological situations 45,46 . The method can also be easily extended to the segmentation of other biological structures of interest, such as the overall cell shapes, as long as the manually segmented datasets is implemented.Other clarification methods specially optimized for thick samples $15,47,49$ could then be also tested with the same global approach presented in this article. The most promising result lay in the possibility of digital clearing. In this study we performed it, for a first demonstration, by training directly on the best clearing method. Variants could be considered like style transfer 50,51 or domain adaptation to further improve the digital clearing of the samples. Also, we demonstrated the possibility of digital clearing by transfer learning even when the two microscopes used are not strictly identical. This calls for a systematic quantitative study to assess the robustness of this finding with microscopes of various resolutions and aberrations. 


\section{Materials and Methods}

We present the details of all the steps described in the graphical abstract of Figure 1.

\section{Sample preparation and image acquisition}

\section{Cell lines and cell culture}

Colorectal carcinoma cell line HCT-116 were used in this study. Cells were cultured in DMEM-GlutaMAX, supplemented with $10 \%$ of Heat-Inactivated Fetal Bovine Serum (FBS; Sigma, St. Louis, Missouri, US), 100 units / $100 \mu \mathrm{g}$ of penicillin / streptomycin and passaged every 3-4 days.

\section{Agarose-based micro-systems}

The micro-systems consist of arrays of 130 micro-wells of $200 \mu m$ in diameter and height. They are used to create 130 reproducible spheroids per condition. This micro-system was produced by moulding agarose (Standard Agarose, $2 \% \mathrm{w} / \mathrm{v}$ ) on PDMS molds created by photolithography process. A patent has been deposited for this process (FR 1870349).

\section{Spheroid formation}

Cells were seeded in each 24 wells-plate containing microsystems at a density of $1,2.10^{5}$ cells $/ \mathrm{mL}, 1 \mathrm{~mL}$ per well. They were placed under orbital agitation (160 rpm) for 2 hours in the incubator $\left(37^{\circ} \mathrm{C}, 5 \% \mathrm{CO} 2\right)$ to increase cell sedimentation inside each micro-well. After 2 hours, the wells were rinsed with warm culture medium $(3 \times)$ to get rid of cells that were out of the micro-wells. The 24 well-plate containing the microsystems were then kept in the incubator. Multicellular Tumor Spheroids (MCTs) are formed within 1 day and are used at Day 5 in this study.

\section{Immunostaining}

At day 5, cells were washed 3 times with warmed PBS for 5 min, followed by paraformaldehyde fixation ( $3.7 \%$ in PBS) for 20 minutes. All wells were then washed with $\mathrm{PBS} / 3 \%$ BSA $(3 \times 5 \mathrm{~min})$, permeabilized with $0.5 \%$ Triton for $20 \mathrm{~min}$, and rinsed again with PBS/ 3\% BSA (3x 5 min). To stain nucleus, NucGreen Dead 488 ReadyProbes Reagent were used (Invitrogen R37109, 2 drops/mL, i.e. 2 drops per wells, 4 hours at room temperature). The samples were washed with PBS $(1 \times 10 \mathrm{~min})$ and kept protected from light in PBS at $4^{\circ} \mathrm{C}$ until image acquisition. Such non-cleared samples were either imaged directly (Control), or further processed with different clearing methods, described below.

\section{Clarification}

Different clarification techniques were used. For RapiClear-clarified samples, the microwells were incubated in RapiClear 1.52 solution (sunjinlab) overnight, then transferred in $0.5 \mathrm{~mm}$ Ispacer (sunjinlab, 2 spacers) and immerged in $35 \mu L$ of fresh RapiClear solution. Sealing was achieved using an additional sticky Ispacer and a coverslip. For Glycerol-clarified samples, the microwells were incubated in a $80 \%$ glycerol solution in PBS (v/v) overnight, then transferred in $0.5 \mathrm{~mm}$ Ispacer (sunjinlab, 2 spacers) and immerged in $35 \mu L$ of fresh $80 \%$ glycerol solution. The chemical compound 2,2'-thiodiethanol (TDE) was also tested in this study, following the procedure described in 52 . Briefly, the microwells were first incubated in a $20 \%$ TDE 
solution in PBS (v/v) for 1 hour, then transferred in a 47\% TDE solution in PBS (v/v) for 2 hours. The microwells were finally transferred in $0.5 \mathrm{~mm}$ Ispacer (sunjinlab, 2 spacers) and immerged in $35 \mu \mathrm{L}$ of fresh $47 \%$ TDE/PBS solution. For ScaleS and CUBIC clarification methods, we used existing dataset of the literature described in 23,44 .

\section{Image acquisition}

A pile of z-stacks images of $1024 \times 1024$ pixels in xy was acquired using a classical confocal set-up. RapiClear images were acquired with Leica SP5 in resonant mode, a 20x dry objective lens $(\mathrm{NA}=0.7)$ is used with a pixel scale of $0.2 \mu \mathrm{m}$ in xy and z-step $1 \mu \mathrm{m}$. Control, Glycerol and TDE images are acquired with Nikon A1Rplus using a 20x water immersion objective lens $(\mathrm{NA}=0.7)$ with a pixel scale of $0.243 \mu \mathrm{m}$ in xy and z-step $2 \mu \mathrm{m}$. A $488 \mathrm{~nm}$ line of an Argon Laser was used to detect NucGreen. Note that, all 2D spheroid images were normalized to have zero mean and variance equal one to compensate for intensity variation before models training process.

\section{Dataset annotation}

Ground truth annotation was performed in semi-automatic way using the interactive learning and segmentation toolkit (Ilastik) 53 based on shallow learning followed by expert correction (Figure 1.c). 2D frames from the beginning, middle and the end of 3D image stacks belonging to each clearing condition were selected for exhaustive representation of instances and clearing effects on image depths. Pixel wise classification mode was used then to generate primary segmentation maps. The filters used in the segmentation step were intensity, edge and textural filters with various kernel sizes ranging from 0.7 pixel to 10 pixels in order to represent the smallest and largest details of the transition between the nuclei and the background (36 filters). Then, we applied the batch processing to segment all 2D data set images. Finally, a manual correction step produced by an expert was applied to the segmentation maps. The overall amount of annotated 2D images was 57 for each clearing conditions. Datasets were then split into 47 for training, 5 for validation and 5 for testing. The total number of cell nuclei was around 10000 for each condition dataset.

3D spheroid images of various thicknesses were normalized in z-depth by considering the slice with the largest diameter as the center of spheroid. Diameter of each spheroid slice was computed from the binarized maximum intensity projection of the $3 \mathrm{D}$ volume on y-axis. The percentage of diameter was derived then for each slice according to the maximum diameter value. Based on this normalization method, and for a fair comparison between the protocols, we considered slices of depths ranging from $70 \%$ to $100 \%$ of maximum diameter (Figure 2 A). It is to be noticed that this limited range of depth causes no loss of information since spheroid are isotropic samples. The missing part of the spheroids are similar to the first half.

\section{Image quality assessment based local metrics}

We first assess locally the quality of spheroid images according to z-depth by using statistical metrics are the signal to noise ratio (SNR) and the contrast to noise ratio (CNR) calculated by two ways: Bhattacharyya coefficient (BC) and Fisher ratio (FR) defined as

$$
\begin{gathered}
S N R=\frac{\mu_{\text {signal }}}{\sigma_{\text {noise }}} \\
C N R_{B C}=1-\sum_{X=1}^{255} \sqrt{p_{d}(X) \cdot q_{d}(X)}
\end{gathered}
$$




$$
C N R_{F R}=\frac{\left(\mu_{\text {signal }}-\mu_{\text {noise }}\right)^{2}}{V_{\text {signal }}+V_{\text {noise }}}
$$

where, $\mu_{\text {signal }}, p_{d}(X)$ and $V_{\text {signal }}$ are the mean intensity of the signal, the probability of a signal intensity value $X$ and the variance of the signal respectively. Also, $\mu_{\text {noise }}, q_{d}(X), V_{\text {noise }}$, and $\sigma_{\text {noise }}$ are the mean intensity of the noise, the probability of a noise intensity value $X$, the variance and the standard deviation of the noise respectively (see SI Figure 1). We computed also the normalized average intensity (average I/Imax) in each image slice to illustrate the change in intensity along the depths. All these factors were computed for three spheroids from each clearing condition.

\section{Deep learning segmentation methods}

To assess the quality of clearing, we tested a set of state-of-the-art segmentation methods based on deep learning that were used for 2D spheroid images segmentation (Figure 1.D). They are briefly presented in this subsection.

\section{U-Net}

We used the reference segmentation network UNet [54] to predict 2 output classes (Cell nuclei and background). We simplified the architecture by reducing the number of feature channels. We use 5 blocks for contracting and expansive paths, each consisting of 2 convolutional layers with $4.2^{n}(n=2,3,4,5,6)$ filters of size $3 \times 3$ and ReLU activation function. For the output probability map $(\hat{y})$, we use a single-channel convolutional layer with sigmoid activation function (see SI Figure 6.A). The total number of trainable parameters was 2, 158, 417 optimized by using the Adam optimizer [55] and the training hyper-parameters are: batch size $=1$, epochs $=33$ and the learning rate $l r=1 e^{-3}$. The final segmentation map was computed by thresholding the probability map by a threshold value $\alpha$ that was optimized on validation data set for each condition. The optimization process is described later in the same section.

\section{Post processing based Dynamic Morphology (DM)}

The same U-Net architecture described previously was applied to predict the probability map $(\hat{y})$ followed by a post processing step based on dynamic morphology [56] and watershed algorithm 57]. This combination was used in histopathology images segmentation to separate touching nuclei [58] that are considered as one object after thresholding the probability map. Briefly, the post processing step was based mainly on the hypothesis that the posterior probability at the border of the touching nuclei was systematically lower than in the putative center of the nucleus, and that the nuclei centers correspond to local maximum intensity in the image. The significant drop of the signal between nuclei center and the border was defined by morphological dynamics as the following: Let $L M$ be a local maximum of the U-Net probability map output $\hat{y}$. $L M$ represents a cell nuclei if along all paths $P$ connecting $L M$ with some higher maximum $L M^{\prime}$, the decrease in $\hat{y}$ is at least $\lambda$

$$
\min _{\substack{P=\left(L M, \ldots, L M^{\prime}\right) \\ \hat{y}\left(L M^{\prime}\right)>\hat{y}(L M)}}\left\{\max _{x \in P}[\hat{y}(L M)-\hat{y}(x)]\right\}>\lambda
$$

where $x$ is a pixel at $(i, j)$ coordinates and $\lambda$ is a free parameter optimized for each clearing method (see SI Figure 6.B. The final segmentation map was then obtained by applying watershed transformation to the inverted probability map seeded from the maxima that fulfill this criterion. 


\section{Dist}

The problem in high nuclei density 2D spheroid images is that the touching and overlapping nuclei are segmented as one object. Several works were proposed to solve this problem by predicting both the object and their contours 59 60]. Others proposed to focus the attention of the model on the core of the nuclei by predicting an eroded version of the annotation as centers correspond to the ultimate erosion of the ground truth 61,62. In our work and unlike pixel-wise binary classification used previously, we follow the proposed work in 37 and we turned 2D spheroid images classification to a regression problem by predicting the distance maps $(\hat{d})$ that focus on the center of nuclei (see SI Figure 6.A). Therefore, for each pixel $x=(i, j)$ of the annotated spheroid binary image $(y)$, with $y(x)>0$, we assign a distance transform $\left(D_{c}\right)$ representing the distance to the closest background pixel $x_{b}=\left[i_{b}, j_{b}\right]$. Here, we used the Chebyshev distance defined as,

$$
D_{c}=\max \left(\left|i_{b}-i\right|, j_{b}-j \mid\right) \text {. }
$$

The Dist model is based on the same U-Net architecture described before. The same training hyper-parameters were used to predict distance maps. Only sigmoid output function was replaced with ReLU function at the output channel since the predicted values are higher than 1 . The final binary segmentation map was then obtained by thresholding the distance output maps $\hat{d}$. The threshold value denoted $\beta$ was also optimized for RapiClear, Control and Glycerol clearing methods. Finally, the post processing step described before was also applied to the predicted maps to enhance the final segmentation after optimizing the parameter $\lambda$ for each clearing method.

\section{Stardist: Star-convex polygons}

Star-convex polygons (Stardist) is one of the robust widely used algorithms for cells detection and segmentation in 2D microscopy images 36. It consists in predicting a star-convex polygon for each cell nuclei pixel $x=[i, j]$ by regressing the distances $\left\{r_{i, j}^{k}\right\}_{k=1}^{\eta}$ of the pixel to the boundary of the nuclei to which belong, along a set of optimized number of radial directions $\eta$ with equidistant angles. Also, separately, the algorithm predicts probability map $\left(p_{i, j}\right)$ for each pixel $x$ as the normalized Euclidean distance to the nearest background pixel $x_{b}=\left[i_{b}, j_{b}\right]$ (see SI Figure 6.A. Given such polygon candidates with their associated nuclei probabilities, a non-maximum suppression (NMS) was performed to reach the final set of polygons, each representing a cell nuclei. Stardist was mainly based on Unet architecture with 3 blocks for contracting and expansive paths, each consisting of 2 convolutional layers with $32.2^{n}(n=0,1,2)$

filters of size $3 \times 3$, and an additional layer of $1283 \times 3$ filters added after the final Unet feature layer to avoid that the subsequent two output layers have to fight over features. The activation functions between layers are ReLU and the total number of trainable parameters was approximately 1.4 million parameters. The output layers of the architecture consist of a single-channel convolutional layer with sigmoid activation for the nuclei probability output and the polygon distance output layer has as many channels as there are radial directions $\eta$ and do not use an additional activation function. We used Stardist in our study to segment cell nuclei inside 2D spheroid images. For the three conditions datasets, the training stage was performed with the following primary hyper parameters: batch size $=1$, epochs $=400$ and the learning rate $l r=0.3 e^{-3}$. After that, the best training model that minimized the loss functions (Equations 6, 7) was selected for each condition. So, based on this criterion the hyper parameters for the selected models of RapiClear, Control and Glycerol datasets were $\left(\right.$ epochs $\left.=317, l r=0.75 e^{-4}\right),\left(\right.$ epochs $\left.=282, l r=1.5 e^{-4}\right)$ and $($ epochs $=380$, $\left.l r=0.75 e^{-4}\right)$ respectively. As in the previously described segmentation methods, the 
parameters: number of rays $\eta$, threshold $\alpha$ of the nuclei probability map and threshold of the non-maximum suppression $\tau$, were optimized for each data set.

\section{Training loss functions and segmentation evaluation metrics}

\section{Loss function}

The purpose of loss function in a deep learning training stage model is to quantify the difference between predictions and ground truths for steering the training of the network. In our work, we used two commonly used loss functions the binary cross entropy $(B C E)$ and the mean squared error $(M S E)$ for classification and regression problem respectively. The $B C E$ for binary classification (nuclei and background) was defined as

$$
B C E(y, \hat{y})=\frac{-1}{M} \sum_{m=1}^{M} y_{m} \cdot \log \left(\hat{y}_{m}\right)+\left(1-y_{m}\right) \cdot \log \left(1-\hat{y}_{m}\right)
$$

and $M S E$ for distance map prediction was defined as,

$$
M S E(d, \hat{d})=\frac{-1}{M} \sum_{m=1}^{M}\left(d_{m}-\hat{d}_{m}\right)^{2}
$$

where $M$ is the $1 D$ output map size, $\hat{y}_{m}$ and $\hat{d}_{m}$ are the $m$-th scalar values in the output predicted maps, $y_{m}$ and $d_{m}$ the corresponding target values of probability maps and distance maps respectively.

\section{Evaluation metrics}

To evaluate the performance of spheroid images segmentation and to be able to quantitatively compare between the transferability of segmentation models trained on clarification method to other clearing protocols, we used the pixel wise-metric F1-score 63 for segmentation evaluation and also the Aggregated Jaccard Index (AJI) [61 as an object-wise metric for touching nuclei splitting evaluation. The F1 measure is defined as the harmonic mean between recall and precision at the pixel level and it was computed as

$$
\text { F1-score }=2 \cdot \frac{\text { Precision } \times \text { Recall }}{\text { Precision }+ \text { Recall }}
$$

with the Precision $=\frac{T P}{T P+F P}$ and Recall $=\frac{T P}{T P+F N}$, where $T P, F P$ and $F N$ are the true positive, false positive and false negative respectively.

The AJI is an extension of the global Jaccard index, where every ground truth nucleus is first matched to one detected nucleus by maximizing the Jaccard index. The AJI corresponds then to the ratio of the sums of the cardinals of intersection and union of these matched components respectively. In addition, all detected components that do not matched were added to the denominator. More formally [37, AJI can be defined as,

$$
A J I=\frac{\sum_{i=1}^{L}\left|G_{i} \cap S_{k}^{*}(i)\right|}{\sum_{i=1}^{L}\left|G_{i} \cup S_{k}^{*}(i)\right|+\sum_{l \in U}\left|S_{l}\right|}
$$

where $G_{i}$ is a nucleus ground truth of $L$ nuclei in an image, $S$ is all the detected nuclei, $S_{k}^{*}(i)$ is the segmented nucleus associated with $G_{i}$ that maximizes the Jaccard index, i.e. $S_{k}^{*}(i)=\operatorname{argmax}_{k} \mid \frac{\left|G_{i} \cap S_{k}\right|}{\left|G_{i} \cup S_{k}\right|}$ and $U$ is the set of indices of detected nuclei that have not been assigned to any ground truth. 


\section{Parameter Optimization}

One important step in supervised learning with deep learning is the hyperparameters fine tuning. This optimization step was applied to validation data set to maximize the segmentation metrics such as F1-score, AJI or Jaccard index for Stardist segmentation method. In our work, threshold of the probability maps $\alpha$, threshold of predicted distance maps $\beta$, the h-minima value $\lambda$ of the dynamic morpholoy reconstruction post processing step, the numbers of rays of the star-convex polygons $\eta$ and threshold $\tau$ of the non-maxmimum suppression (nms) were optimized empirically by varying each parameter between a range of values (see SI Figure 7). Then, the value that maximized a segmentation metric were selected for each segmentation method depending on the RapiClear, Control and Glycerol segmentation models as shown in Table 2.

\begin{tabular}{|c|c|c|c|c|c|c|}
\hline \multirow{2}{*}{ Parameters } & \multirow{2}{*}{ Segmentation methods } & \multirow{2}{*}{ Ranges } & \multirow{2}{*}{ Metrics } & \multicolumn{3}{|c|}{ Segmentation models } \\
\hline & & & & Control & RapiClear & Glycerol \\
\hline \multirow{2}{*}{$\alpha$} & Unet & $\alpha \in\left[\begin{array}{ll}0 & 1\end{array}\right]$ & F1-score & 0.5 & 0.6 & 0.4 \\
\hline & Stardist & $\alpha \in\left[\begin{array}{ll}0.3 & 0.7\end{array}\right]$ & Jaccard index & 0.6 & 0.4 & 0.5 \\
\hline$\beta$ & Dist & $\beta \in\left[\begin{array}{ll}0 & 2\end{array}\right]$ & F1-score & 1.1 & 1.2 & 0.6 \\
\hline \multirow{2}{*}{$\lambda$} & Unet + DM & \multirow{2}{*}{$\lambda \in\left[\begin{array}{ll}0 & 20\end{array}\right]$} & \multirow{2}{*}{ AJI } & 8 & 7 & 2 \\
\hline & Dist + DM & & & 2 & 1 & 1 \\
\hline$\eta$ & \multirow{2}{*}{ Stardist } & $\eta \in 2^{i}, i \in[28]$ & F1-score & \multicolumn{3}{|c|}{64} \\
\hline$\tau$ & & $\tau \in\left[\begin{array}{ll}0.3 & 0.5\end{array}\right]$ & Jaccard index & 0.4 & 0.3 & 0.4 \\
\hline
\end{tabular}

Table 2. Parameters optimization step. Various thresholds were optimized for each used segmentation method between a range of tested values. The value of threshold that maximized a metric was selected.

\section{Transfer learning}

We used transfer learning [64] methodology in our study to transfer knowledge gained from training segmentation model on a dataset from a clarification condition and apply the pre-trained model to segment images clarified from other conditions. This was done by brute transfer of the weights in the neural network without fine tuning.

\section{Supporting information}

\section{S1 Fig. Supplementary Figures 1,2,3,4,5 and 6}

S1 Datasets and Videos. Datasets availability and 3D spheroids reconstruction videos. Annotated data sets used during the current study are available and supplementary videos of 3D reconstructed spheroids for Control (non cleared), Rapiclear and Glycerol cleared samples are available in the following repository: https://uabox.univ-angers.fr/index.php/s/6myuGGs0JO94M8D.

\section{Acknowledgments}

Ali Ahmad gratefully acknowledges project EU H2020 FET Open, PROCHIP, Chromatin organization PROfiling with high- throughput super-resolution microscopy on a CHIP, grant agreement no. 801336 (https://pro-chip.eu/) for funding his PhD. The authors also thank the GdR ImaBio for financial support to the organisation of a deep learning hackathon which contributed to the initiation of this study. 


\section{Author contributions statement}

A.A. and D.R. conceived of and designed the experiments. S.G., G.R. and C.R prepared and acquired the spheroid samples. A.A. annotated the data used in the experiments. A.A conceived and developed image quality assessment and image segmentation based on deep learning algorithms. A.A., D.R. G.R. and C.R analysed the data and the results. All authors contributed to the redaction of the manuscript and approved the final version. D.R. and C.F. supervised the PROCHIP project.

425

\section{References}

1. Sutherland RM. Cell and environment interactions in tumor microregions: the multicell spheroid model. Science. 1988;240(4849):177-184.

2. Nunes AS, Barros AS, Costa EC, Moreira AF, Correia IJ. 3D tumor spheroids as in vitro models to mimic in vivo human solid tumors resistance to therapeutic drugs. Biotechnology and bioengineering. 2019;116(1):206-226.

3. Mittler F, Obeïd P, Rulina AV, Haguet V, Gidrol X, Balakirev MY. High-content monitoring of drug effects in a 3D spheroid model. Frontiers in oncology. $2017 ; 7: 293$.

4. Langhans SA. Three-dimensional in vitro cell culture models in drug discovery and drug repositioning. Frontiers in pharmacology. 2018;9:6.

5. Edmondson R, Broglie JJ, Adcock AF, Yang L. Three-dimensional cell culture systems and their applications in drug discovery and cell-based biosensors. Assay and drug development technologies. 2014;12(4):207-218.

6. Booij TH, Price LS, Danen EH. 3D cell-based assays for drug screens: challenges in imaging, image analysis, and high-content analysis. SLAS DISCOVERY: Advancing Life Sciences R\&D. 2019;24(6):615-627.

7. Mehta G, Hsiao AY, Ingram M, Luker GD, Takayama S. Opportunities and challenges for use of tumor spheroids as models to test drug delivery and efficacy. Journal of controlled release. 2012;164(2):192-204.

8. Dekkers JF, Alieva M, Wellens LM, Ariese HC, Jamieson PR, Vonk AM, et al. High-resolution 3D imaging of fixed and cleared organoids. Nature protocols. 2019;14(6):1756.

9. Silvestri L, Costantini I, Sacconi L, Pavone FS. Clearing of fixed tissue: a review from a microscopist's perspective. Journal of biomedical optics. 2016;21(8):081205.

10. Chen YY, Silva PN, Syed AM, Sindhwani S, Rocheleau JV, Chan WC. Clarifying intact 3D tissues on a microfluidic chip for high-throughput structural analysis. Proceedings of the National Academy of Sciences. 2016;113(52):14915-14920.

11. Santisteban TS, Rabajania O, Kalinina I, Robinson S, Meier M. Rapid spheroid clearing on a microfluidic chip. Lab on a Chip. 2018;18(1):153-161.

12. Costa EC, Silva DN, Moreira AF, Correia IJ. Optical clearing methods: An overview of the techniques used for the imaging of 3D spheroids. Biotechnology and bioengineering. 2019;116(10):2742-2763. 
13. Oliveira LMC, Tuchin VV. The Optical Clearing Method: A New Tool for Clinical Practice and Biomedical Engineering. Springer Nature; 2019.

14. Boutin ME, Hoffman-Kim D. Application and assessment of optical clearing methods for imaging of tissue-engineered neural stem cell spheres. Tissue Engineering Part C: Methods. 2015;21(3):292-302.

15. Nürnberg E, Vitacolonna M, Klicks J, von Molitor E, Cesetti T, Keller F, et al. Routine Optical Clearing of 3D-Cell Cultures: Simplicity Forward. Frontiers in Molecular Biosciences. 2020;7:20.

16. Wan P, Zhu J, Xu J, Li Y, Yu T, Zhu D. Evaluation of seven optical clearing methods in mouse brain. Neurophotonics. 2018;5(3):035007.

17. Sirenko O, Mitlo T, Hesley J, Luke S, Owens W, Cromwell EF. High-content assays for characterizing the viability and morphology of $3 \mathrm{D}$ cancer spheroid cultures. Assay and drug development technologies. 2015;13(7):402-414.

18. Chan LLY, Cribbes S, Kessel S, McMenemy S, Qiu J. A high-throughput 3D tumor spheroid screening method for drug discovery using imaging cytometry; 2017.

19. Ekert JE, Johnson K, Strake B, Pardinas J, Jarantow S, Perkinson R, et al. Three-dimensional lung tumor microenvironment modulates therapeutic compound responsiveness in vitro-implication for drug development. PloS one. 2014;9(3):e92248.

20. Chen W, Wong C, Vosburgh E, Levine AJ, Foran DJ, Xu EY. High-throughput image analysis of tumor spheroids: a user-friendly software application to measure the size of spheroids automatically and accurately. Journal of Visualized Experiments (JoVE). 2014;(89):e51639.

21. Vinci M, Gowan S, Boxall F, Patterson L, Zimmermann M, Lomas C, et al. Advances in establishment and analysis of three-dimensional tumor spheroid-based functional assays for target validation and drug evaluation. BMC biology. 2012;10(1):29.

22. Alsehli H, Mosis F, Thomson C, Hamrud E, Wiseman E, Gentleman E, et al. An integrated pipeline for high-throughput screening and profiling of spheroids using simple live image analysis of frame to frame variations. Methods. 2020;

23. Boutin ME, Voss TC, Titus SA, Cruz-Gutierrez K, Michael S, Ferrer M. A high-throughput imaging and nuclear segmentation analysis protocol for cleared 3D culture models. Scientific reports. 2018;8(1):1-14.

24. Mathew B, Schmitz A, Muñoz-Descalzo S, Ansari N, Pampaloni F, Stelzer EH, et al. Robust and automated three-dimensional segmentation of densely packed cell nuclei in different biological specimens with Lines-of-Sight decomposition. BMC bioinformatics. 2015;16(1):187.

25. Barbier M, Jaensch S, Cornelissen F, Vidic S, Gjerde K, de Hoogt R, et al. Ellipsoid segmentation model for analyzing light-attenuated 3D confocal image stacks of fluorescent multi-cellular spheroids. PloS one. 2016;11(6):e0156942.

26. Dunn KW, Fu C, Ho DJ, Lee S, Han S, Salama P, et al. DeepSynth: Three-dimensional nuclear segmentation of biological images using neural networks trained with synthetic data. Scientific reports. 2019;9(1):1-15. 
27. Tasnadi EA, Toth T, Kovacs M, Diosdi A, Pampaloni F, Molnar J, et al. 3D-Cell-Annotator: an open-source active surface tool for single-cell segmentation in 3D microscopy images. Bioinformatics. 2020;36(9):2948-2949.

28. Piccinini F, Balassa T, Carbonaro A, Diosdi A, Toth T, Moshkov N, et al. Software tools for 3D nuclei segmentation and quantitative analysis in multicellular aggregates. Computational and Structural Biotechnology Journal. $2020 ;$.

29. Kovac B, Fehrenbach J, Guillaume L, Weiss P. FitEllipsoid: a fast supervised ellipsoid segmentation plugin. BMC bioinformatics. 2019;20(1):1-8.

30. Schmitz A, Fischer SC, Mattheyer C, Pampaloni F, Stelzer EH. Multiscale image analysis reveals structural heterogeneity of the cell microenvironment in homotypic spheroids. Scientific reports. 2017;7(1):1-13.

31. Goyal B, Dogra A, Agrawal S, Sohi B, Sharma A. Image denoising review: From classical to state-of-the-art approaches. Information Fusion. 2020;55:220-244.

32. Xing F, Yang L. Robust nucleus/cell detection and segmentation in digital pathology and microscopy images: a comprehensive review. IEEE reviews in biomedical engineering. 2016;9:234-263.

33. Toyoshima Y, Tokunaga T, Hirose O, Kanamori M, Teramoto T, Jang MS, et al. Accurate automatic detection of densely distributed cell nuclei in 3D space. PLoS computational biology. 2016;12(6):e1004970.

34. Weigert M, Schmidt U, Haase R, Sugawara K, Myers G. Star-convex polyhedra for $3 \mathrm{~d}$ object detection and segmentation in microscopy. In: The IEEE Winter Conference on Applications of Computer Vision; 2020. p. 3666-3673.

35. Xie L, Qi J, Pan L, Wali S. Integrating deep convolutional neural networks with marker-controlled watershed for overlapping nuclei segmentation in histopathology images. Neurocomputing. 2020;376:166-179.

36. Schmidt U, Weigert M, Broaddus C, Myers G. Cell detection with star-convex polygons. In: International Conference on Medical Image Computing and Computer-Assisted Intervention. Springer; 2018. p. 265-273.

37. Naylor P, Laé M, Reyal F, Walter T. Segmentation of nuclei in histopathology images by deep regression of the distance map. IEEE transactions on medical imaging. 2018;38(2):448-459.

38. Jung H, Lodhi B, Kang J. An automatic nuclei segmentation method based on deep convolutional neural networks for histopathology images. BMC Biomedical Engineering. 2019;1(1):24.

39. Jimenez-Carretero D, Abrishami V, Fernández-de Manuel L, Palacios I, Quílez-Álvarez A, Díez-Sánchez A, et al. Tox_ (R) CNN: Deep learning-based nuclei profiling tool for drug toxicity screening. PLoS computational biology. 2018;14(11):e1006238.

40. Yang L, Ghosh RP, Franklin JM, Chen S, You C, Narayan RR, et al. NuSeT: A deep learning tool for reliably separating and analyzing crowded cells. PLoS computational biology. 2020;16(9):e1008193. 
41. Kecheril Sadanandan S, Karlsson J, Wahlby C. Spheroid segmentation using multiscale deep adversarial networks. In: Proceedings of the IEEE International Conference on Computer Vision Workshops; 2017. p. 36-41.

42. Khoshdeli M, Winkelmaier G, Parvin B. Multilayer Encoder-Decoder Network for 3D Nuclear Segmentation in Spheroid Models of Human Mammary Epithelial Cell Lines. In: Proceedings of the IEEE Conference on Computer Vision and Pattern Recognition Workshops; 2018. p. 2239-2245.

43. Tan SH, Swathi Y, Tan S, Goh J, Seishima R, Murakami K, et al. AQP5 enriches for stem cells and cancer origins in the distal stomach. Nature. 2020;578(7795):437-443.

44. Masson A, Escande P, Frongia C, Clouvel G, Ducommun B, Lorenzo C. High-resolution in-depth imaging of optically cleared thick samples using an adaptive SPIM. Scientific reports. 2015;5:16898.

45. Drost J, Clevers H. Organoids in cancer research. Nature Reviews Cancer. 2018;18(7):407-418.

46. Dutta D, Heo I, Clevers H. Disease modeling in stem cell-derived 3D organoid systems. Trends in molecular medicine. 2017;23(5):393-410.

47. Lai HM, Liu AKL, Ng HHM, Goldfinger MH, Chau TW, DeFelice J, et al. Next generation histology methods for three-dimensional imaging of fresh and archival human brain tissues. Nature communications. 2018;9(1):1-12.

48. Li W, Germain RN, Gerner MY. Multiplex, quantitative cellular analysis in large tissue volumes with clearing-enhanced 3D microscopy (Ce3D). Proceedings of the National Academy of Sciences. 2017;114(35):E7321-E7330.

49. Zhu J, Yu T, Li Y, Xu J, Qi Y, Yao Y, et al. MACS: Rapid Aqueous Clearing System for 3D Mapping of Intact Organs. Advanced Science. 2020;7(8):1903185.

50. Hollandi R, Szkalisity A, Toth T, Tasnadi E, Molnar C, Mathe B, et al. nucleAIzer: A parameter-free deep learning framework for nucleus segmentation using image style transfer. Cell Systems. 2020;.

51. Cho H, Lim S, Choi G, Min H. Neural stain-style transfer learning using gan for histopathological images. arXiv preprint arXiv:171008543. 2017;.

52. Costantini I, Ghobril JP, Di Giovanna AP, Mascaro ALA, Silvestri L, Müllenbroich MC, et al. A versatile clearing agent for multi-modal brain imaging. Scientific reports. 2015;5:9808.

53. Berg S, Kutra D, Kroeger T, Straehle CN, Kausler BX, Haubold C, et al. ilastik: interactive machine learning for (bio)image analysis. Nature Methods. 2019; p. $1-7$.

54. Ronneberger O, Fischer P, Brox T. U-net: Convolutional networks for biomedical image segmentation. In: International Conference on Medical image computing and computer-assisted intervention. Springer; 2015. p. 234-241.

55. Kingma DP, Ba J. Adam: A method for stochastic optimization. arXiv preprint arXiv:14126980. 2014;.

56. Grimaud M. New measure of contrast: the dynamics. In: Image Algebra and Morphological Image Processing III. vol. 1769. International Society for Optics and Photonics; 1992. p. 292-305. 
57. Beucher S, Meyer F. The morphological approach to segmentation: the watershed transformation. Mathematical morphology in image processing. 1993;34:433-481.

58. Naylor P, Laé M, Reyal F, Walter T. Nuclei segmentation in histopathology images using deep neural networks. In: 2017 IEEE 14th international symposium on biomedical imaging (ISBI 2017). IEEE; 2017. p. 933-936.

59. Kumar N, Verma R, Sharma S, Bhargava S, Vahadane A, Sethi A. A dataset and a technique for generalized nuclear segmentation for computational pathology. IEEE transactions on medical imaging. 2017;36(7):1550-1560.

60. Van Valen DA, Kudo T, Lane KM, Macklin DN, Quach NT, DeFelice MM, et al. Deep learning automates the quantitative analysis of individual cells in live-cell imaging experiments. PLoS computational biology. 2016;12(11).

61. Kumar N, Verma R, Arora A, Kumar A, Gupta S, Sethi A, et al. Convolutional neural networks for prostate cancer recurrence prediction. In: Medical Imaging 2017: Digital Pathology. vol. 10140. International Society for Optics and Photonics; 2017. p. $101400 H$.

62. Sirinukunwattana K, Raza SEA, Tsang YW, Snead DR, Cree IA, Rajpoot NM. Locality sensitive deep learning for detection and classification of nuclei in routine colon cancer histology images. IEEE transactions on medical imaging. 2016;35(5):1196-1206.

63. Chinchor N, Sundheim BM. MUC-5 evaluation metrics. In: Fifth Message Understanding Conference (MUC-5): Proceedings of a Conference Held in Baltimore, Maryland, August 25-27, 1993; 1993.

64. Pan SJ, Yang Q. A survey on transfer learning. IEEE Transactions on knowledge and data engineering. 2009;22(10):1345-1359. 
A.
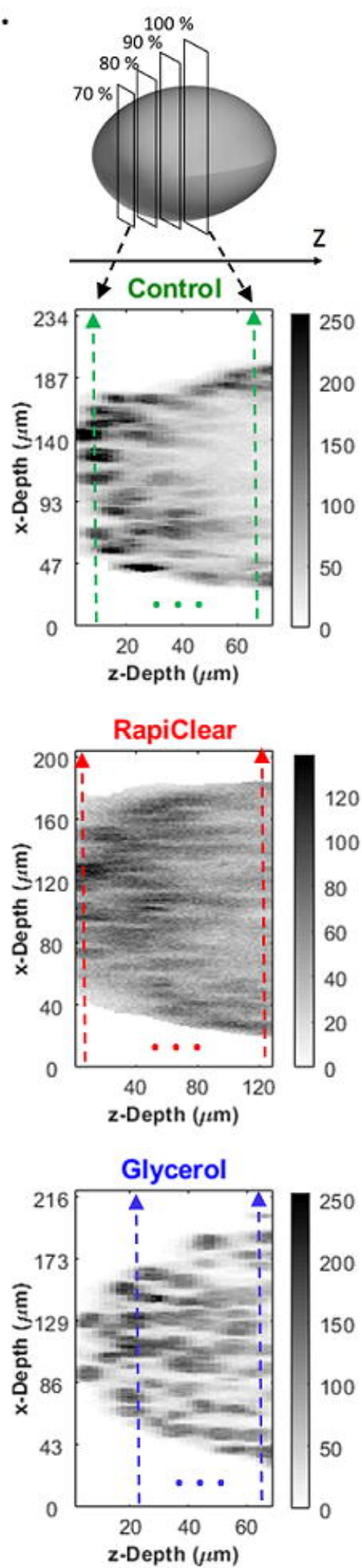

B.
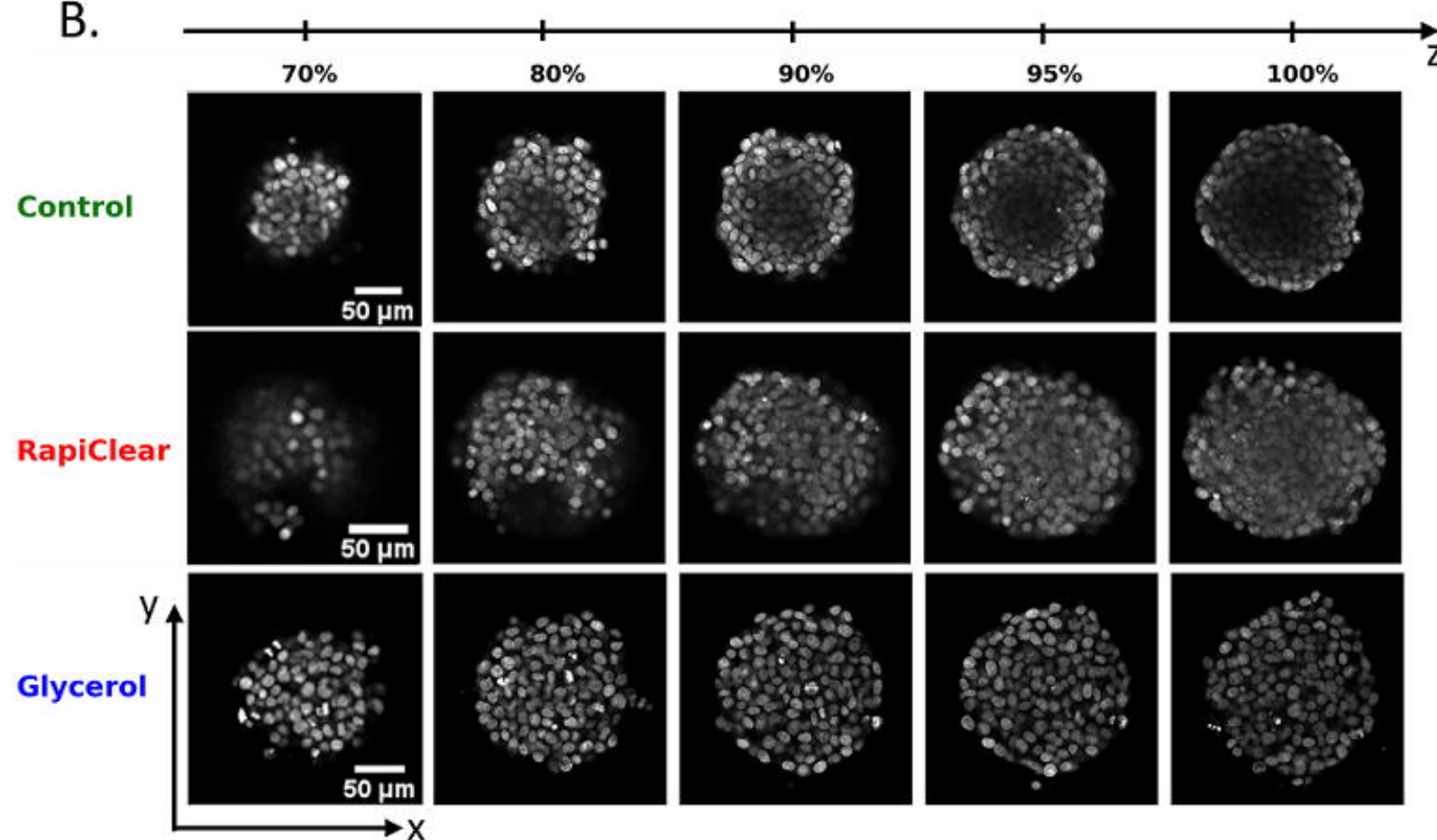

c.
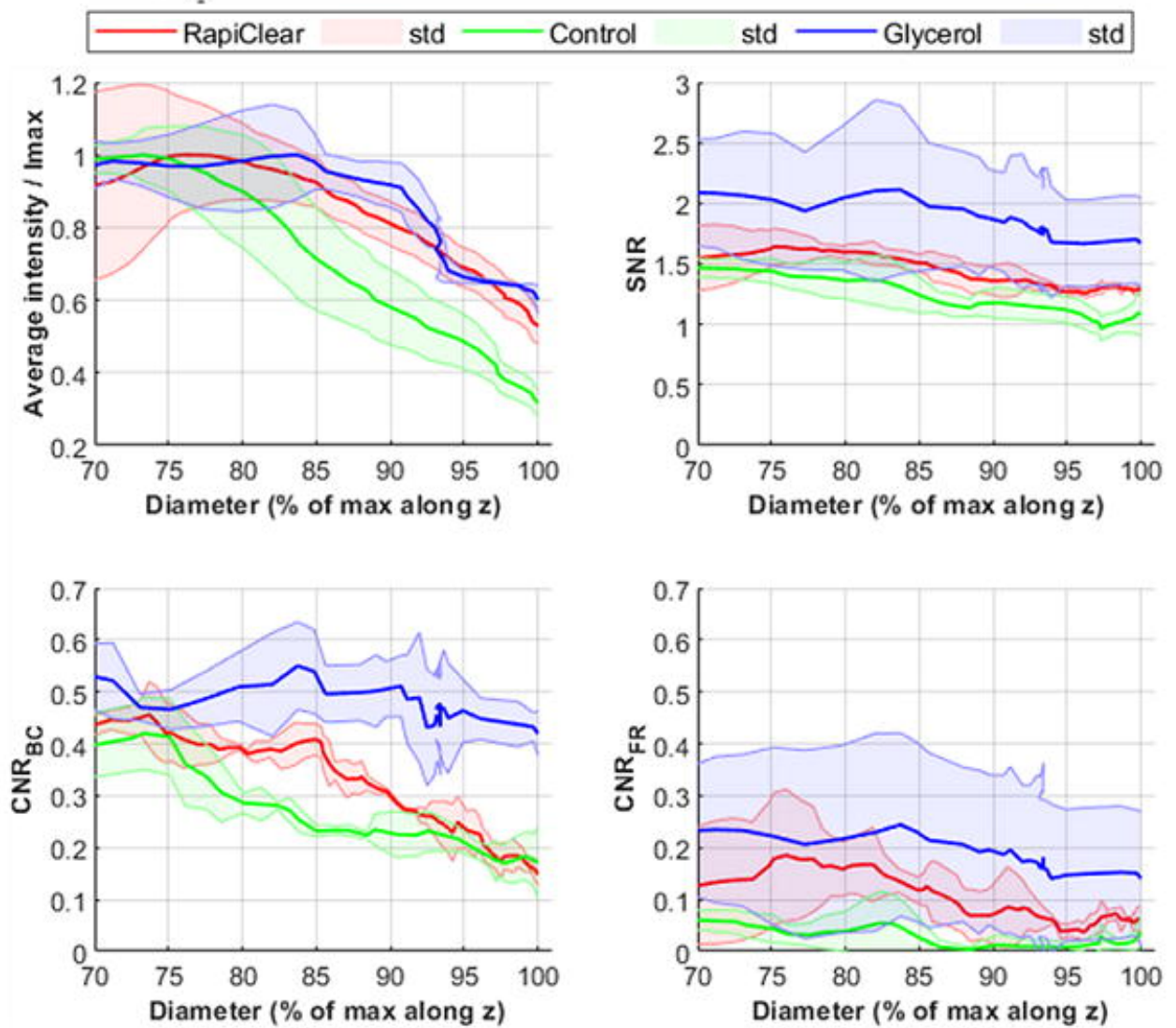
A.
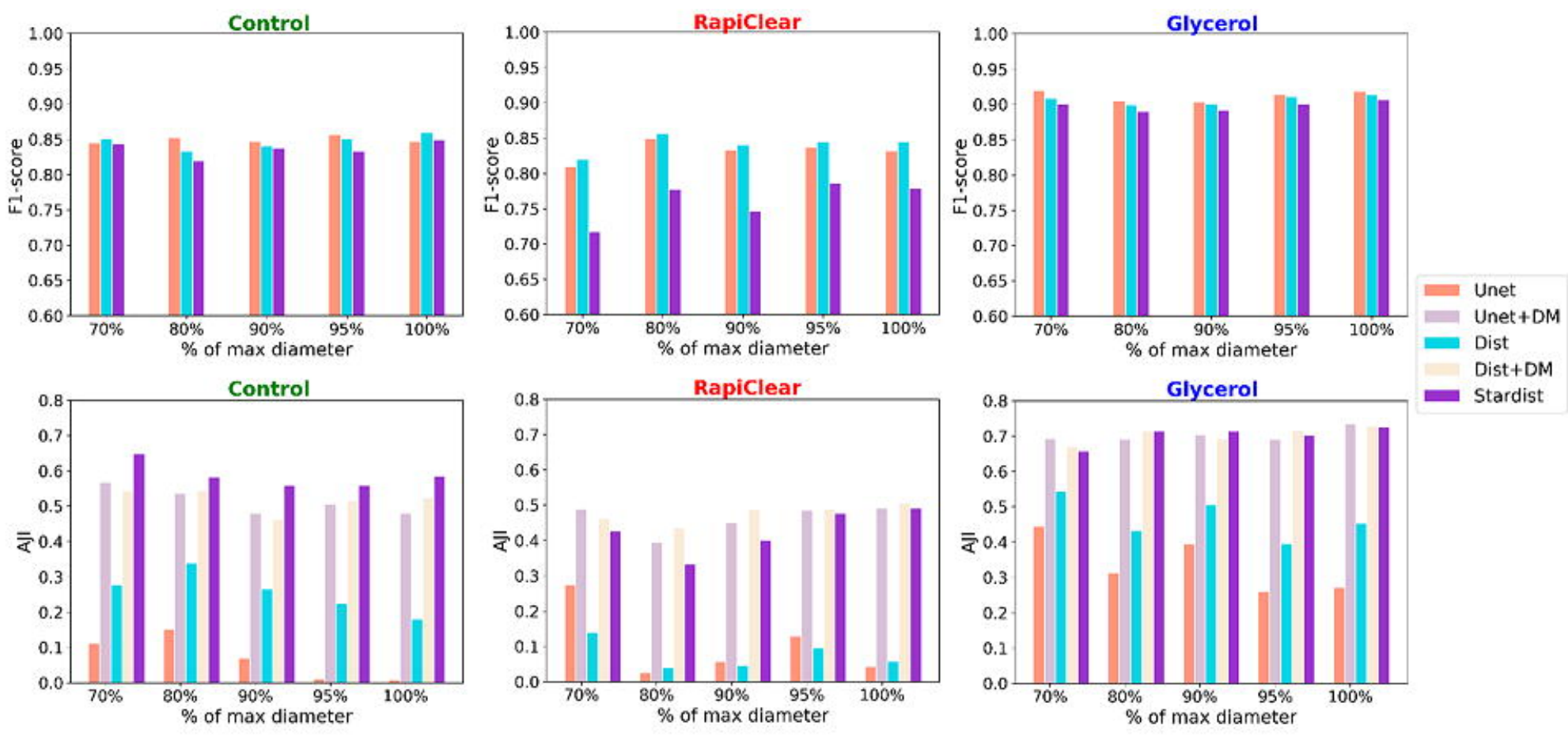

B.

Control

Test images: $90 \%$
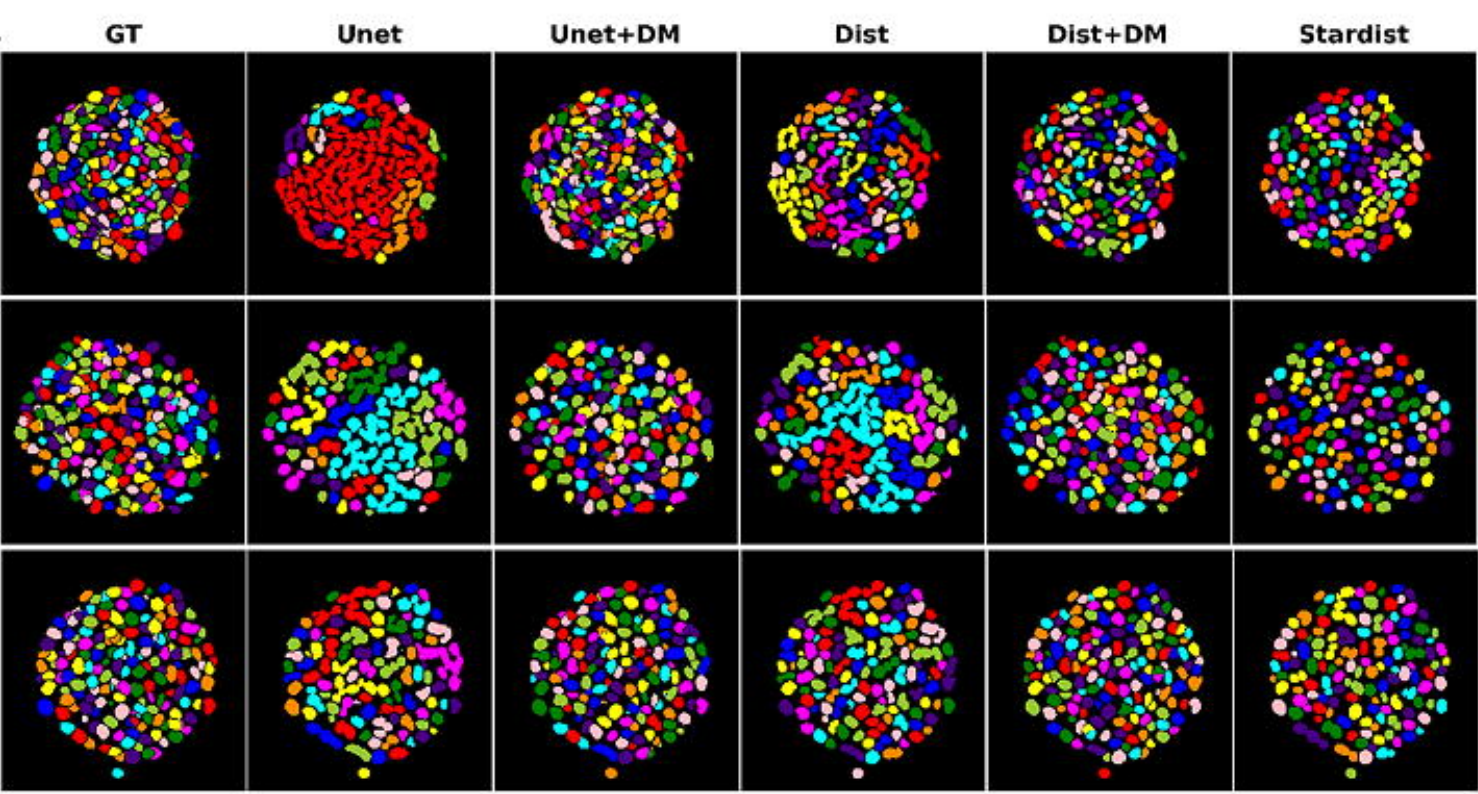

Glycerol
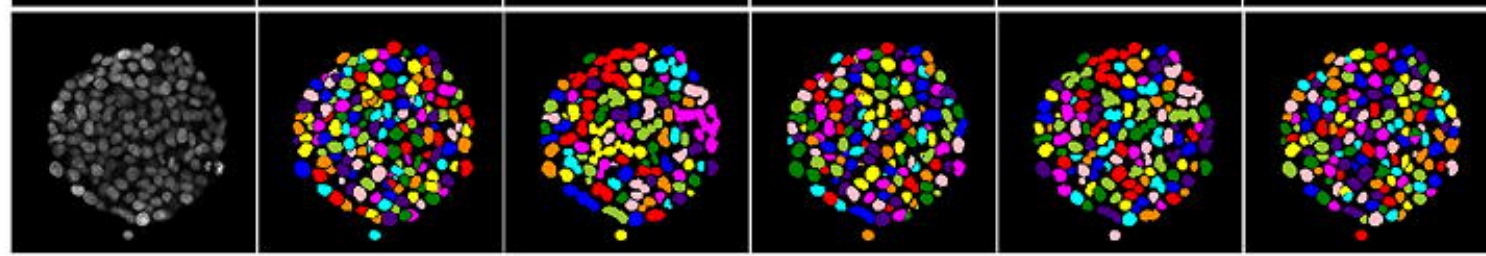
A.
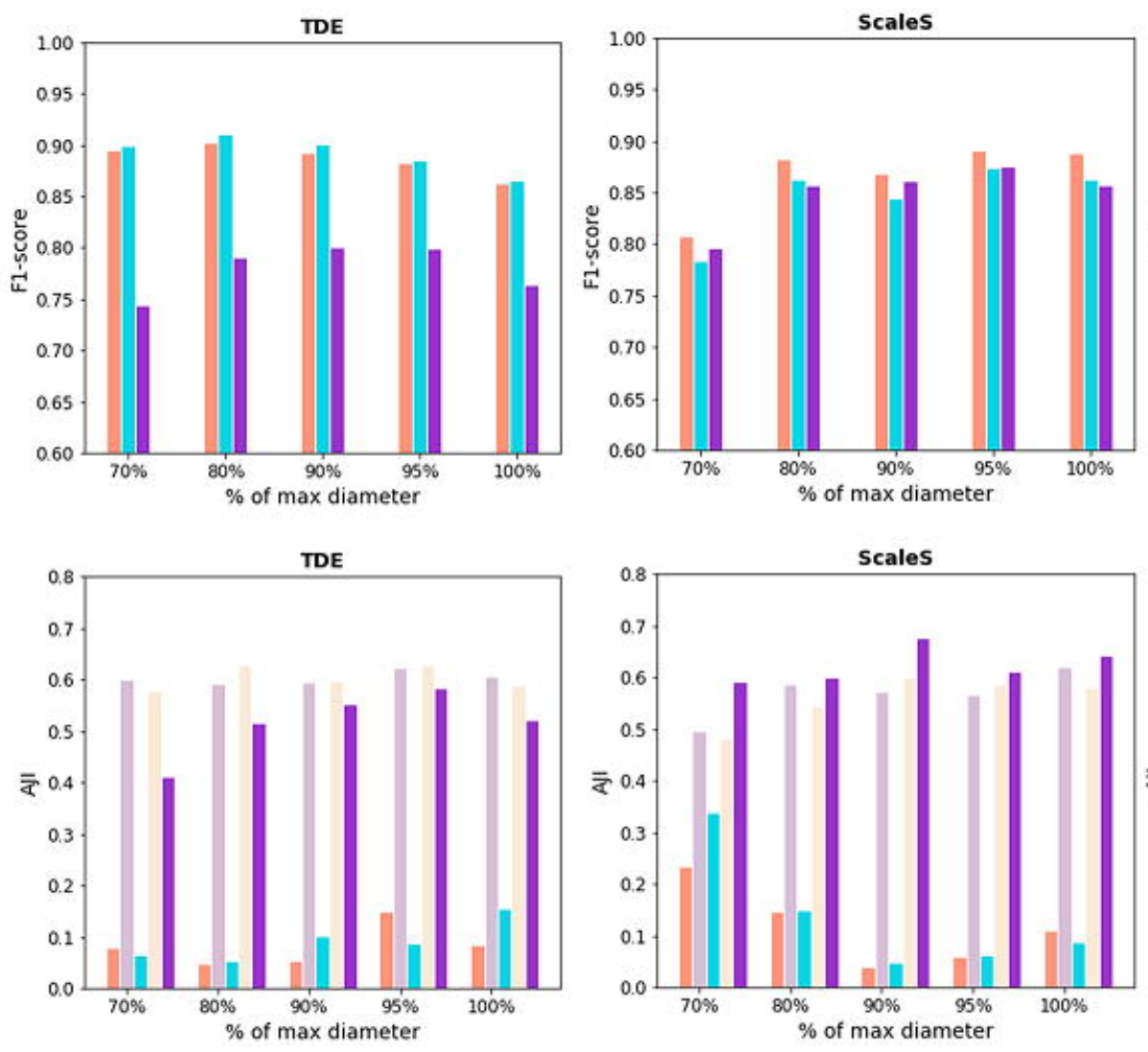
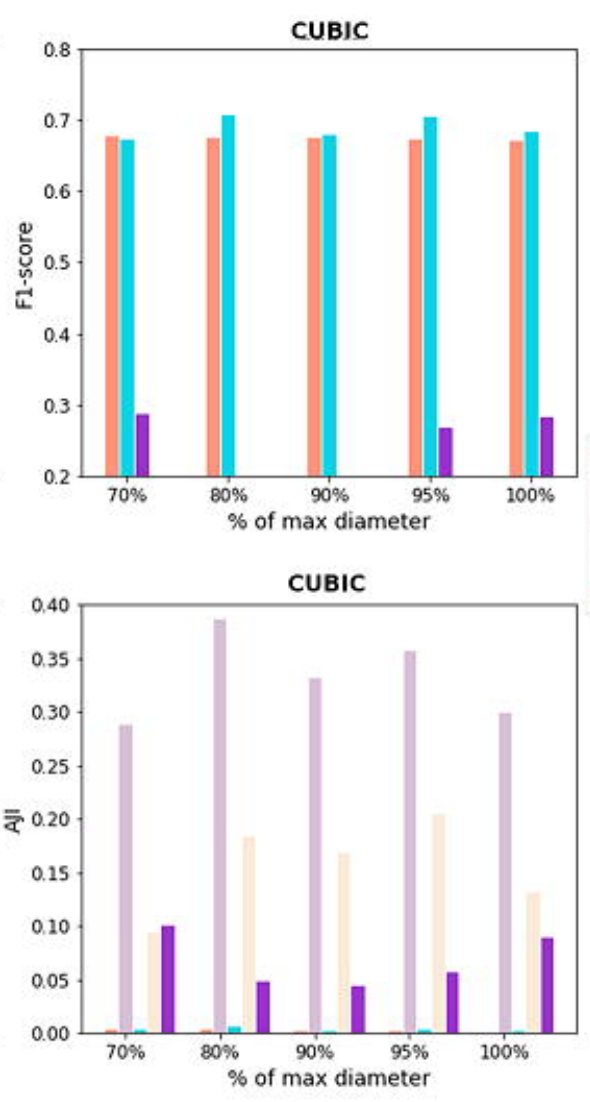

B.

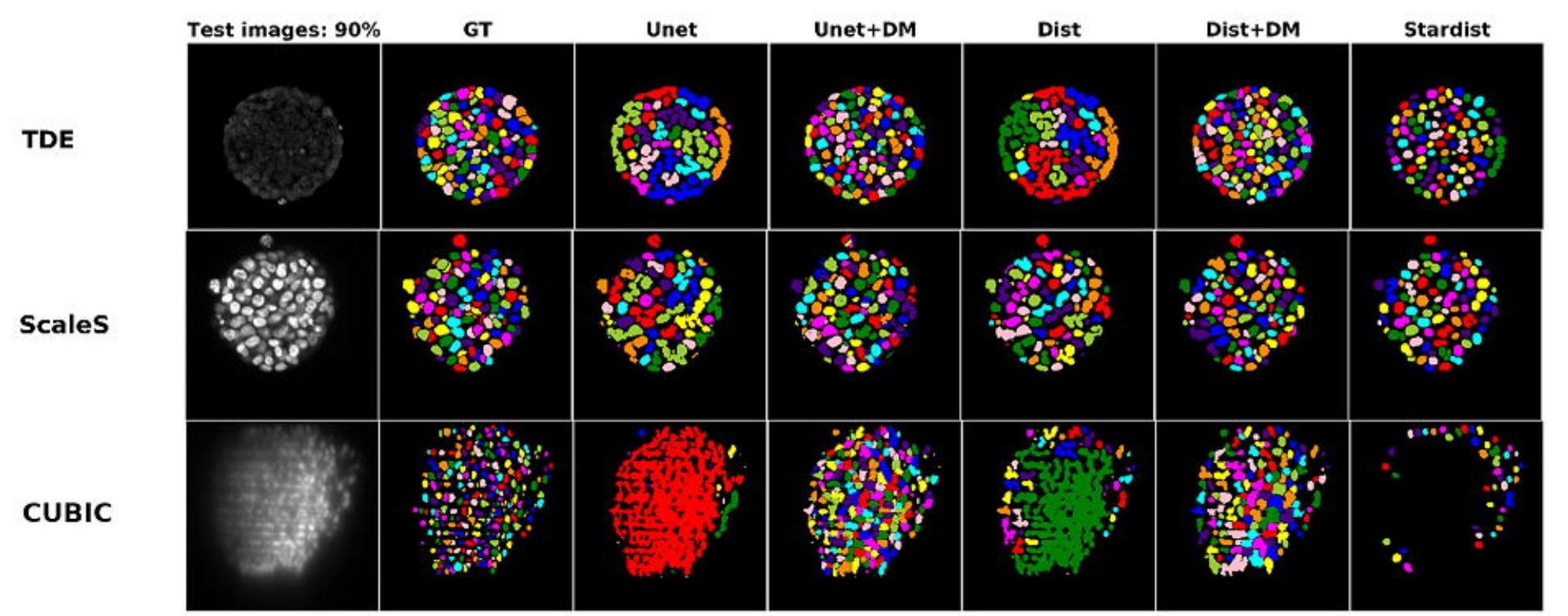


A.

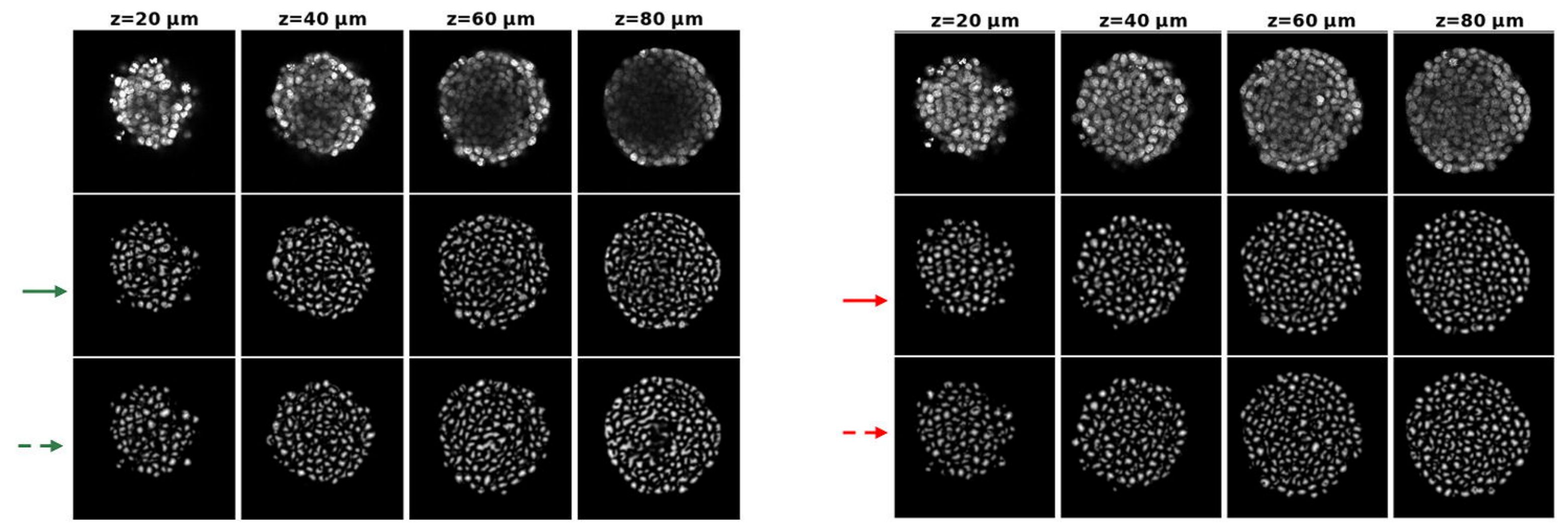

- Control $\quad--$ Control with Digital Clearing $\quad$ Ground truth

B.

_ RapiClear _--_ RapiClear with Digital Clearing
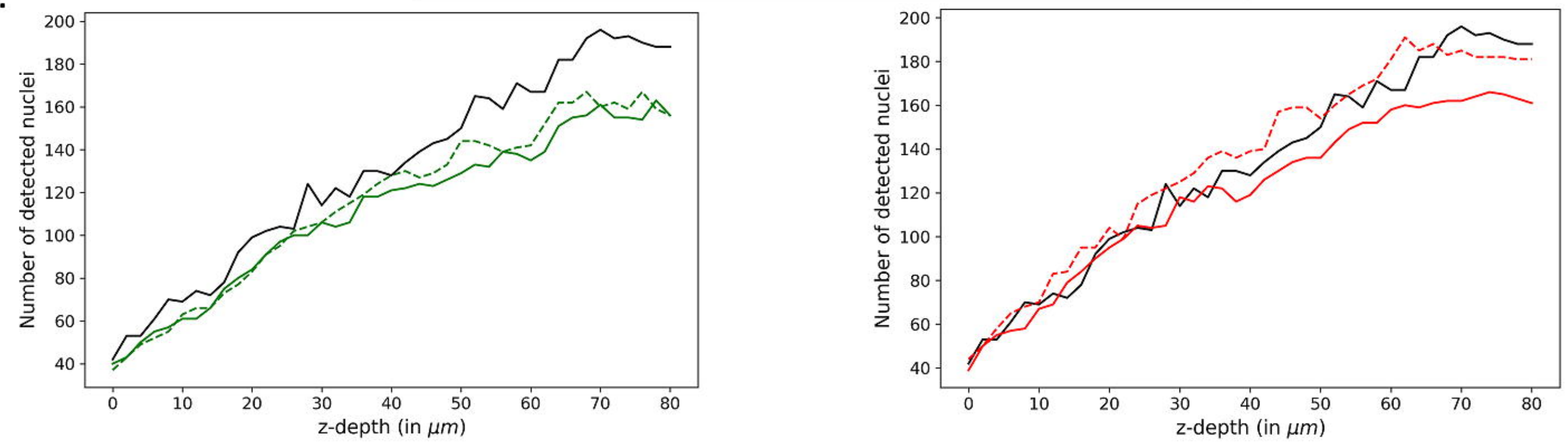
A.

\section{Control}

\section{Patches crop}

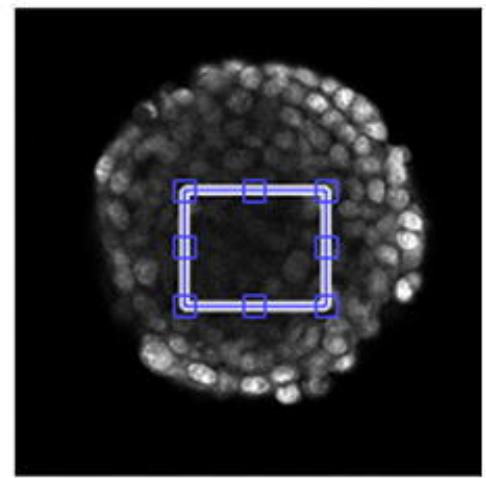

RapiClear

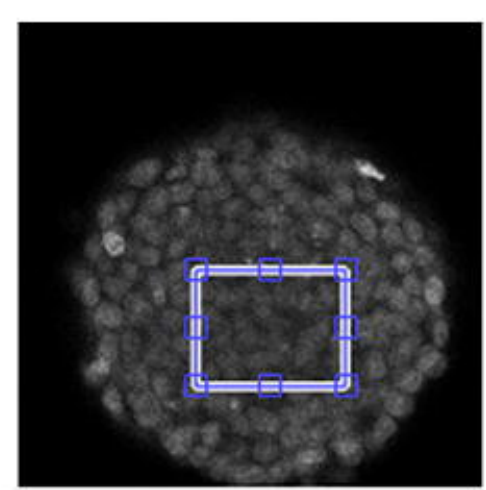

Glycerol

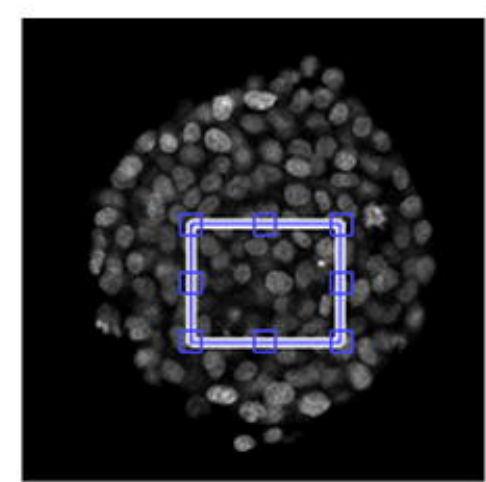

B.
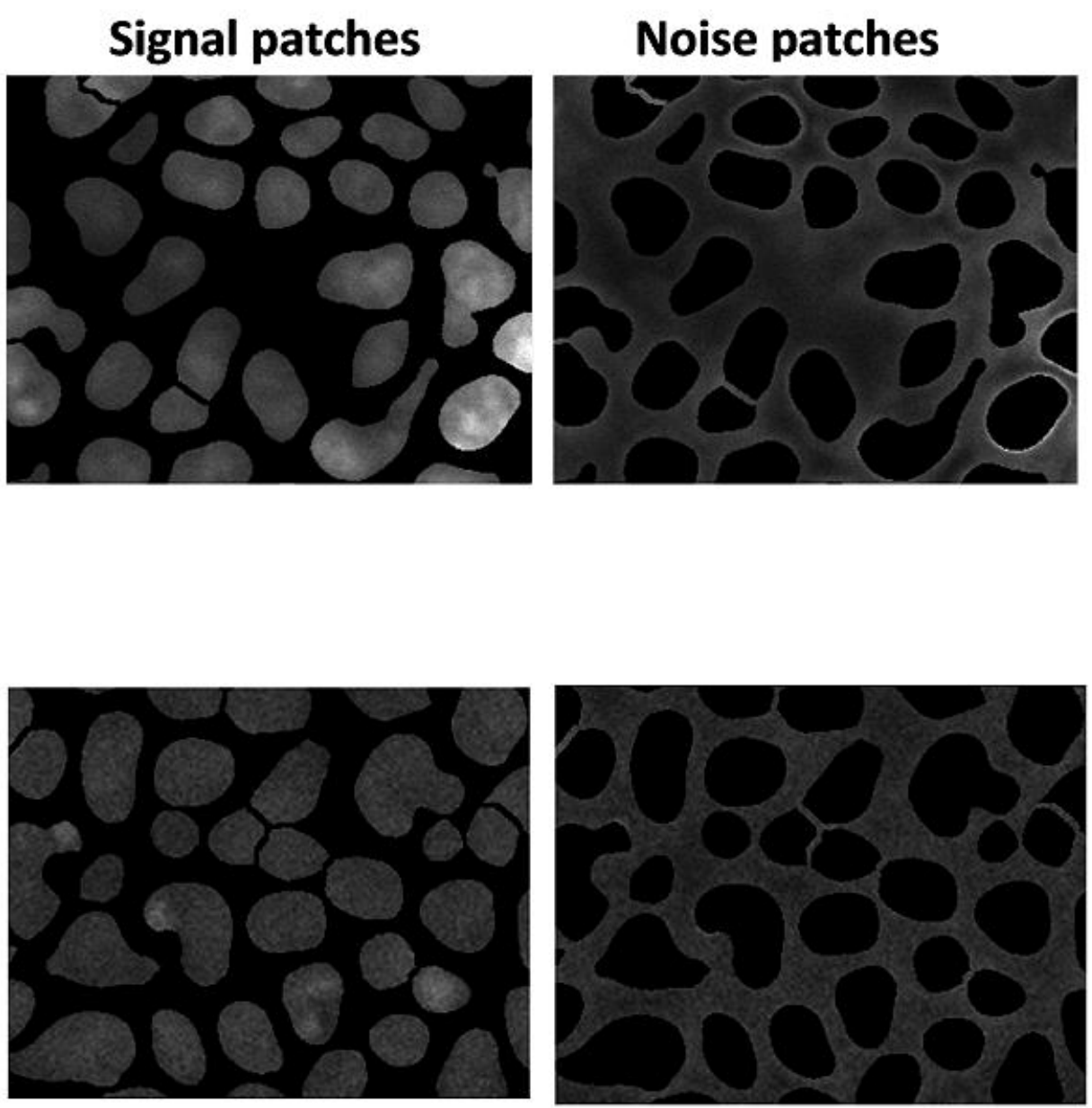

C.
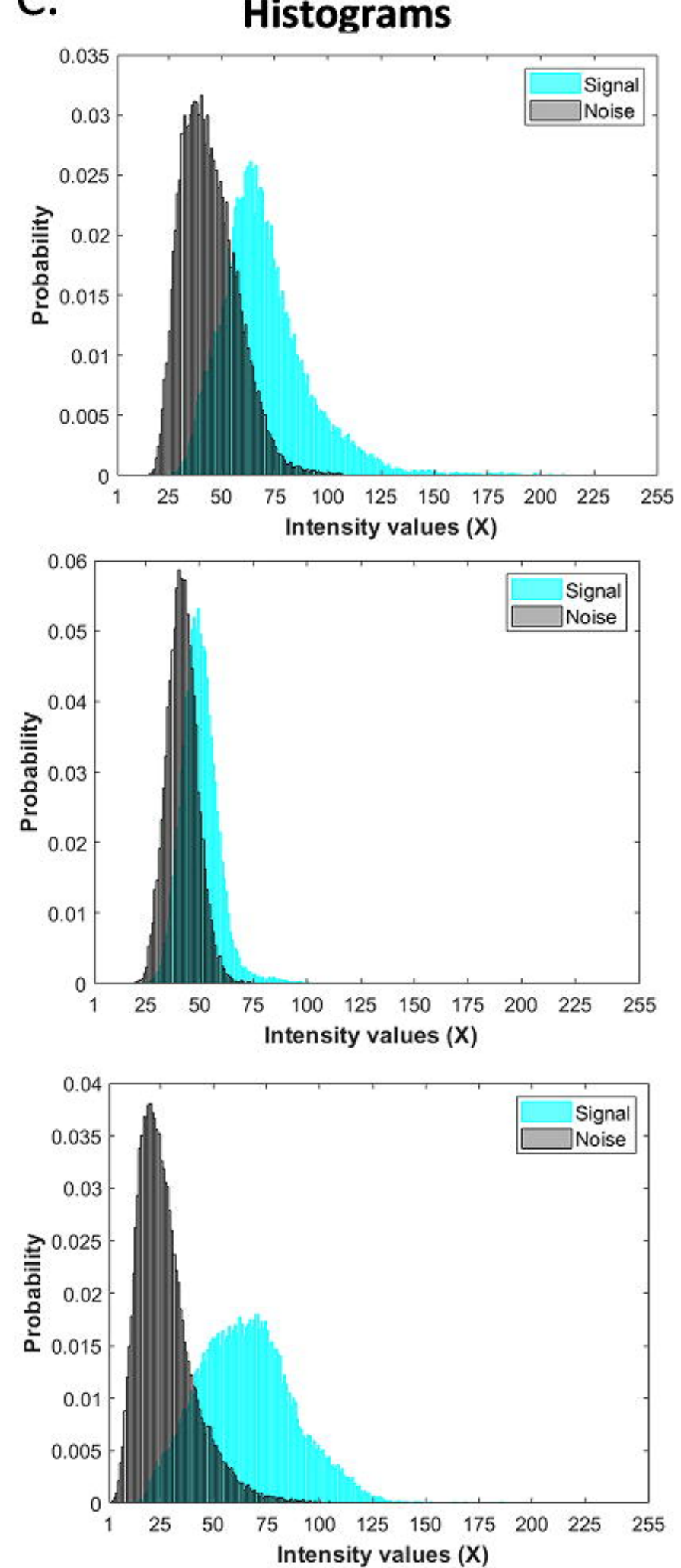

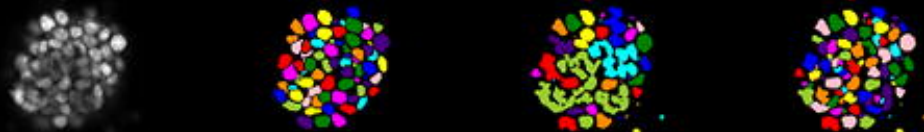



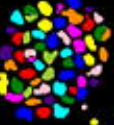

$\frac{108}{80}$

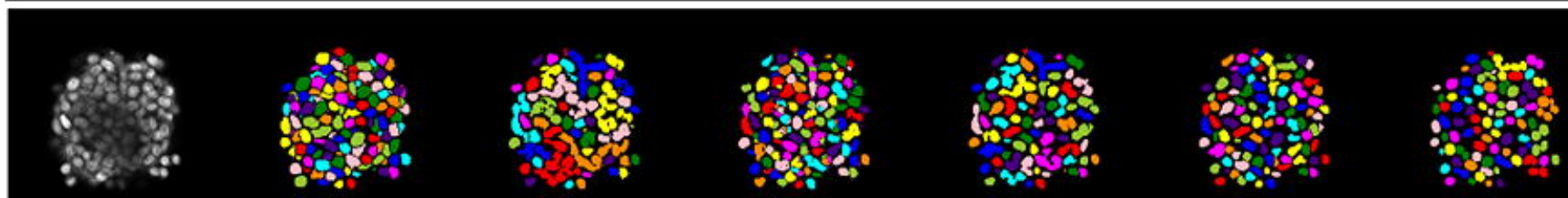
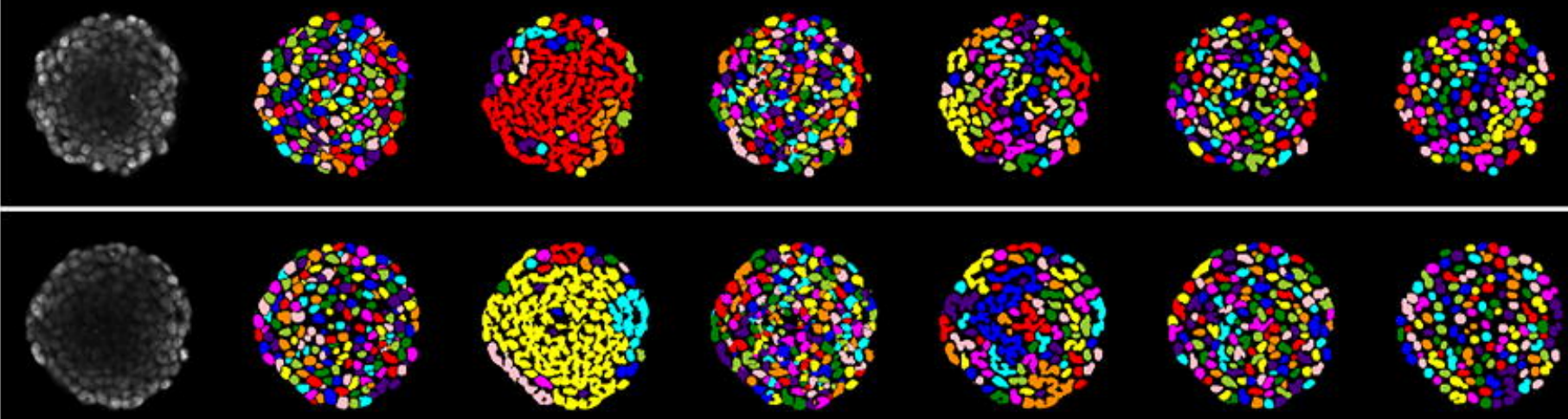

B.

RapiClear
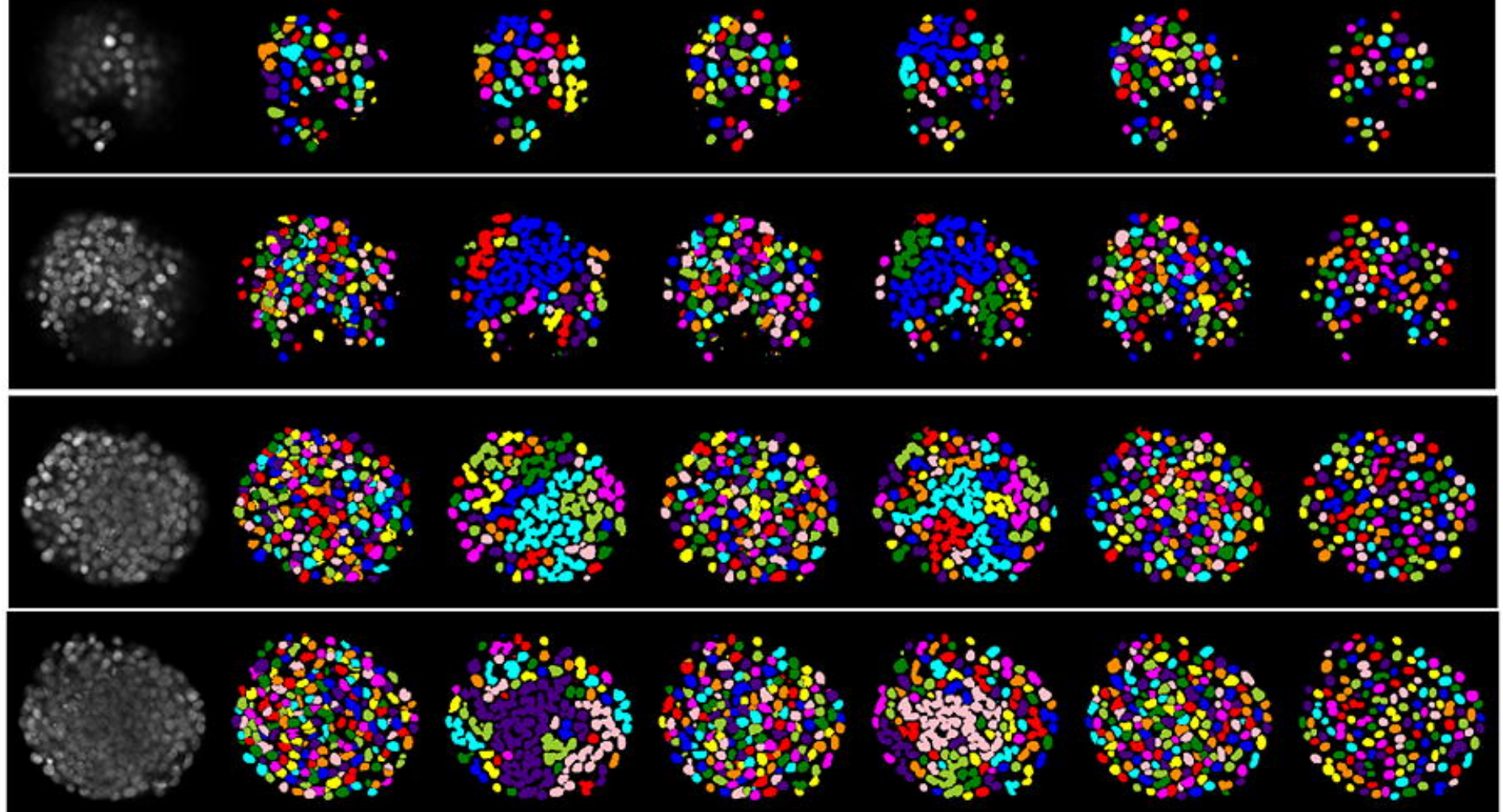
A.

Glycerol

Test images Unet Unet+DM Dist Dist+DM Stardist

3238 03035 $100 \mathrm{~s}$ 80
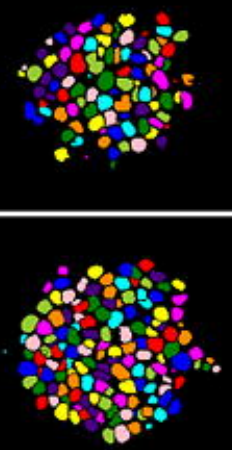

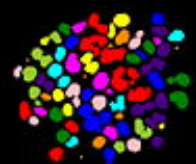

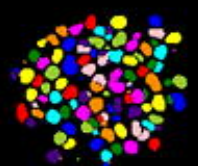

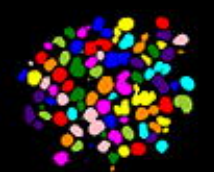

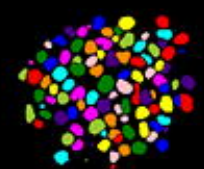

280

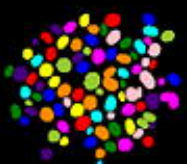

$80 \%$

$95 \%$

$100 \%$

B.

$70 \%$

$80 \%$

$95 \%$

$100 \%$
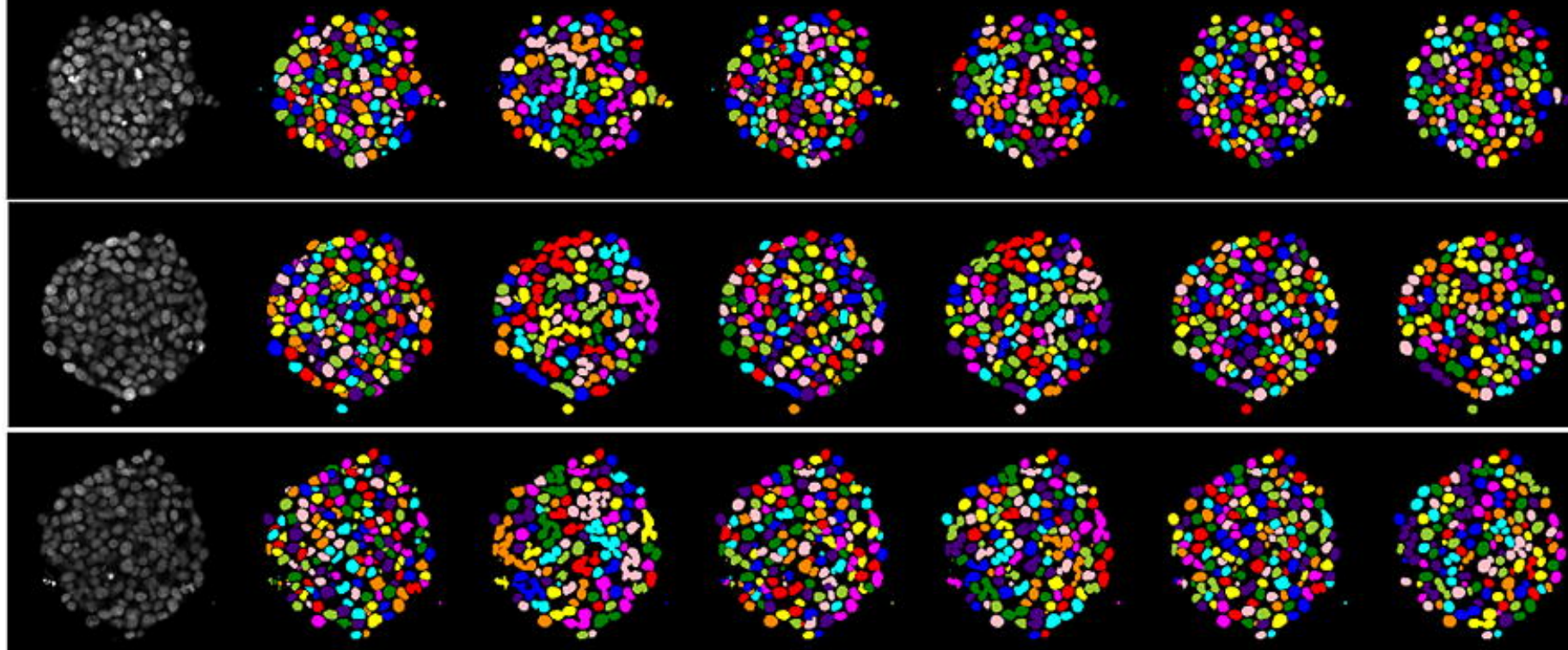

-
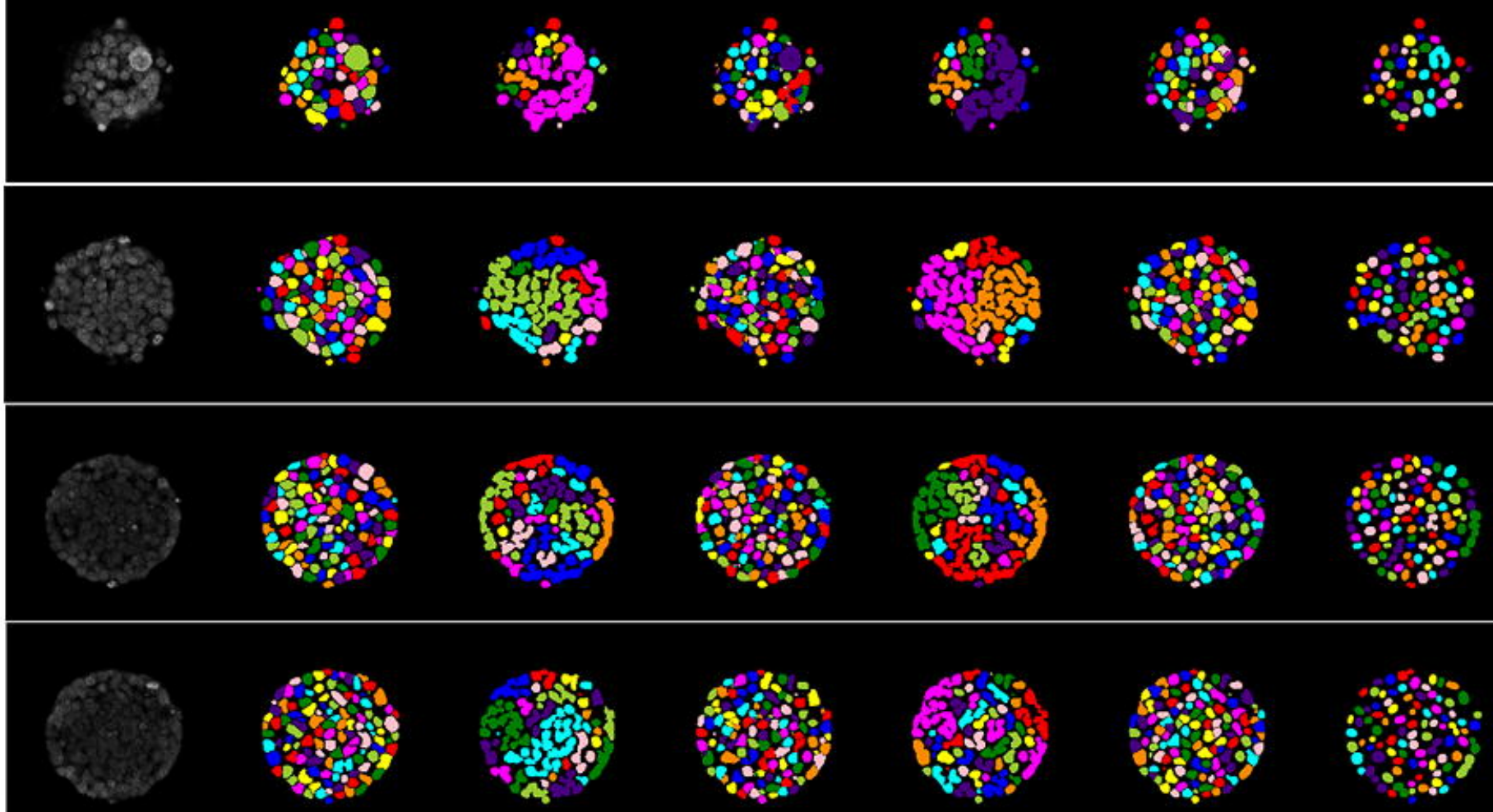

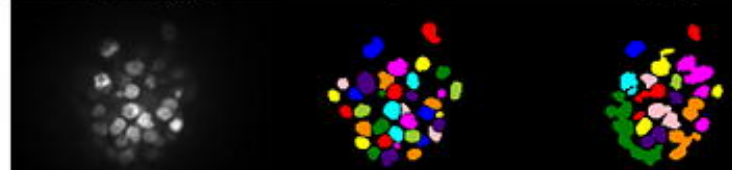

ise.

$\underbrace{323}_{2}$

8.

$88^{\circ}$
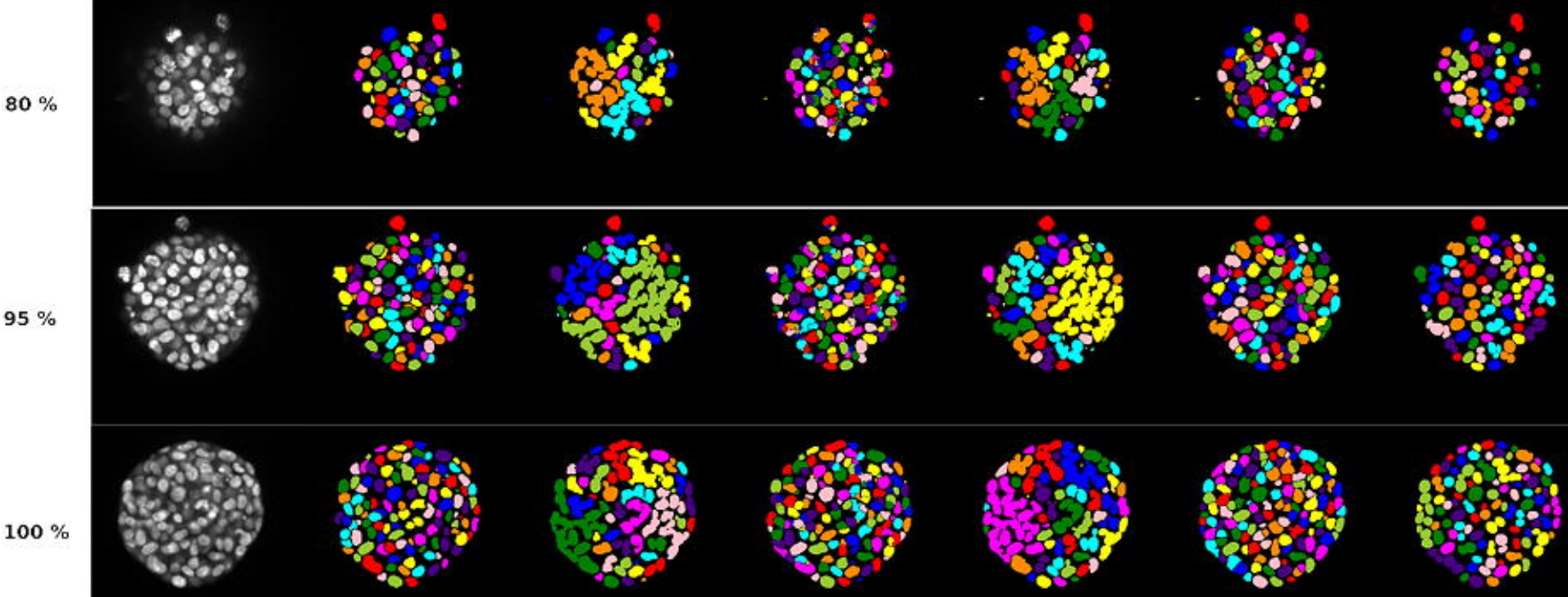

B.

\section{CUBIC}
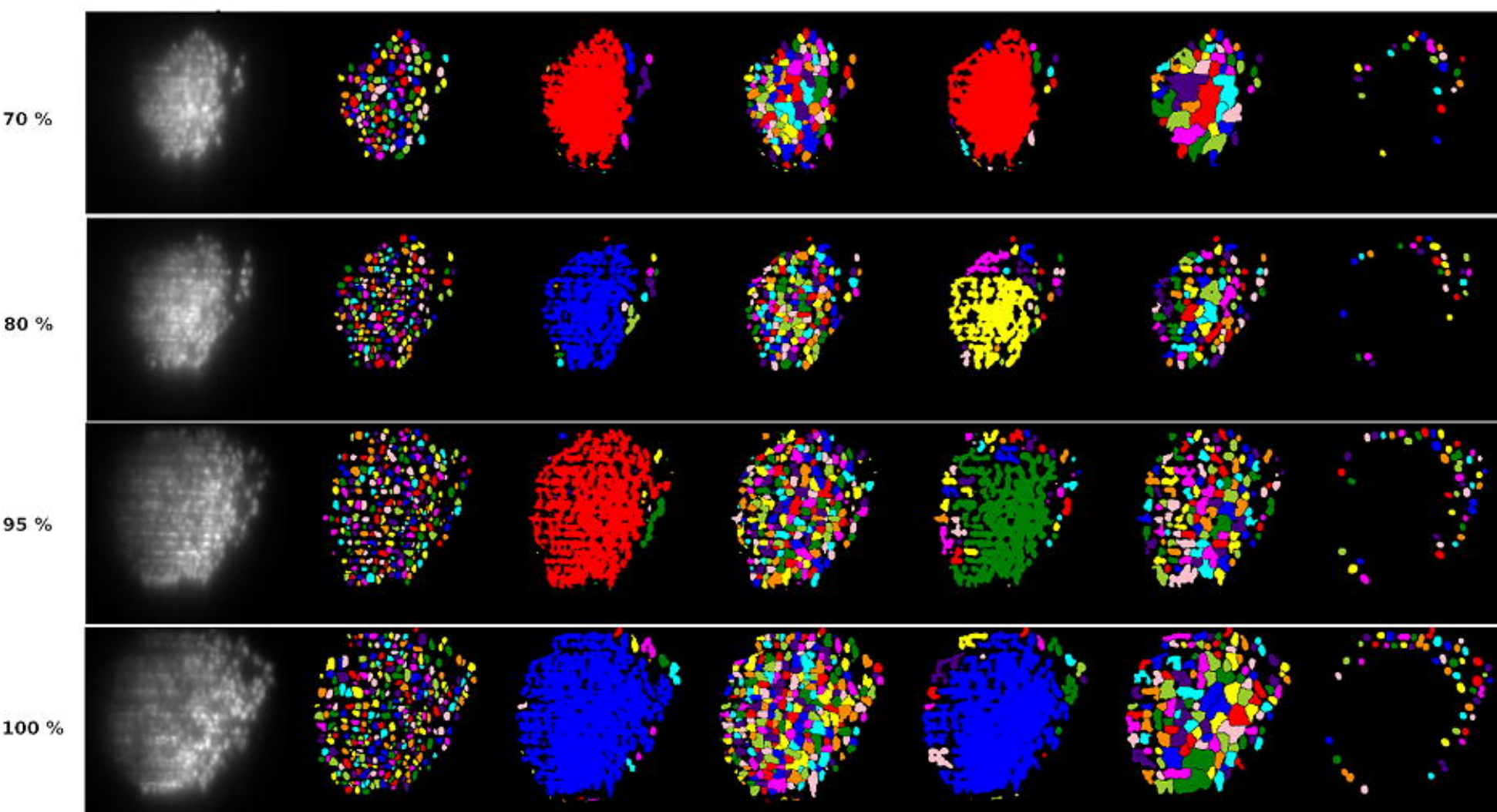


\section{Control}

A.

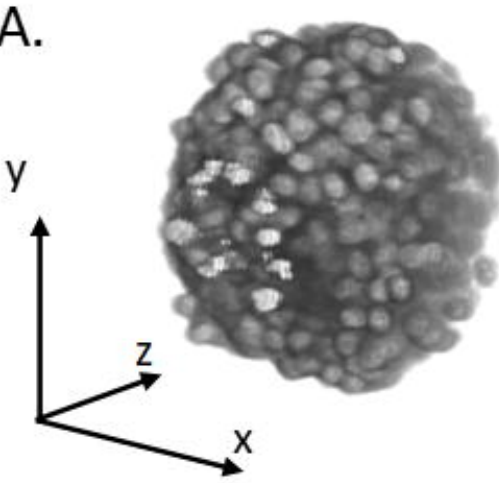

B.

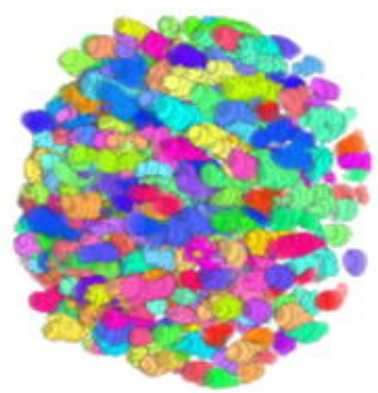

RapiClear

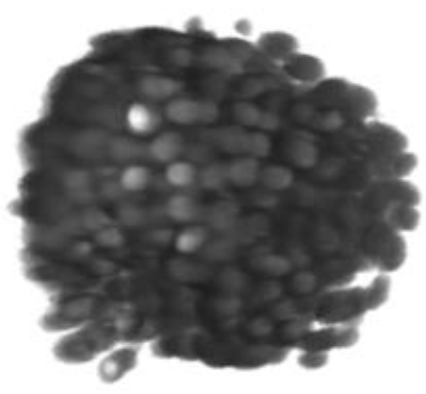

Glycerol
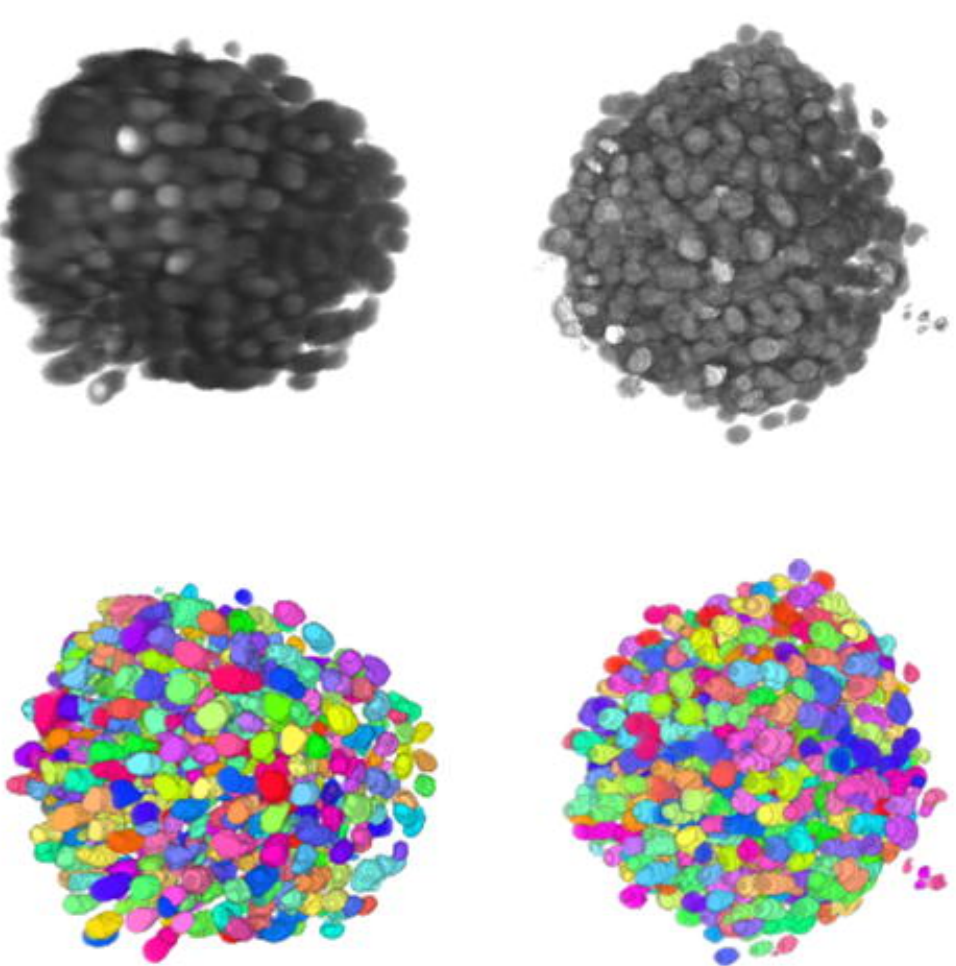
A.
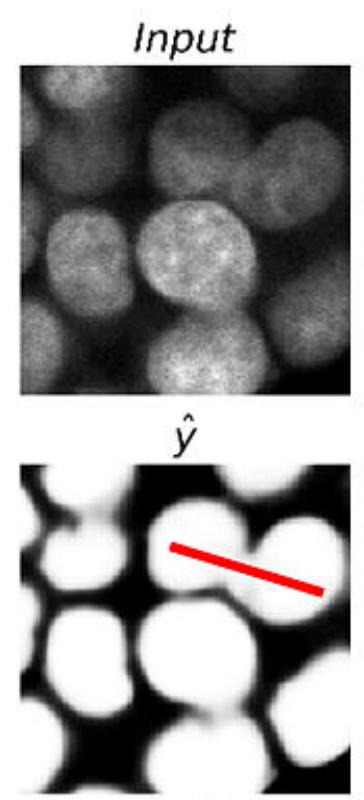

$\left\{r_{i, j}^{k}\right\}_{k=1}^{64}$

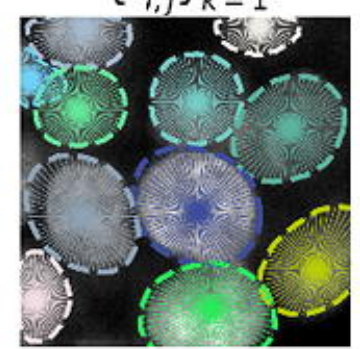

B.

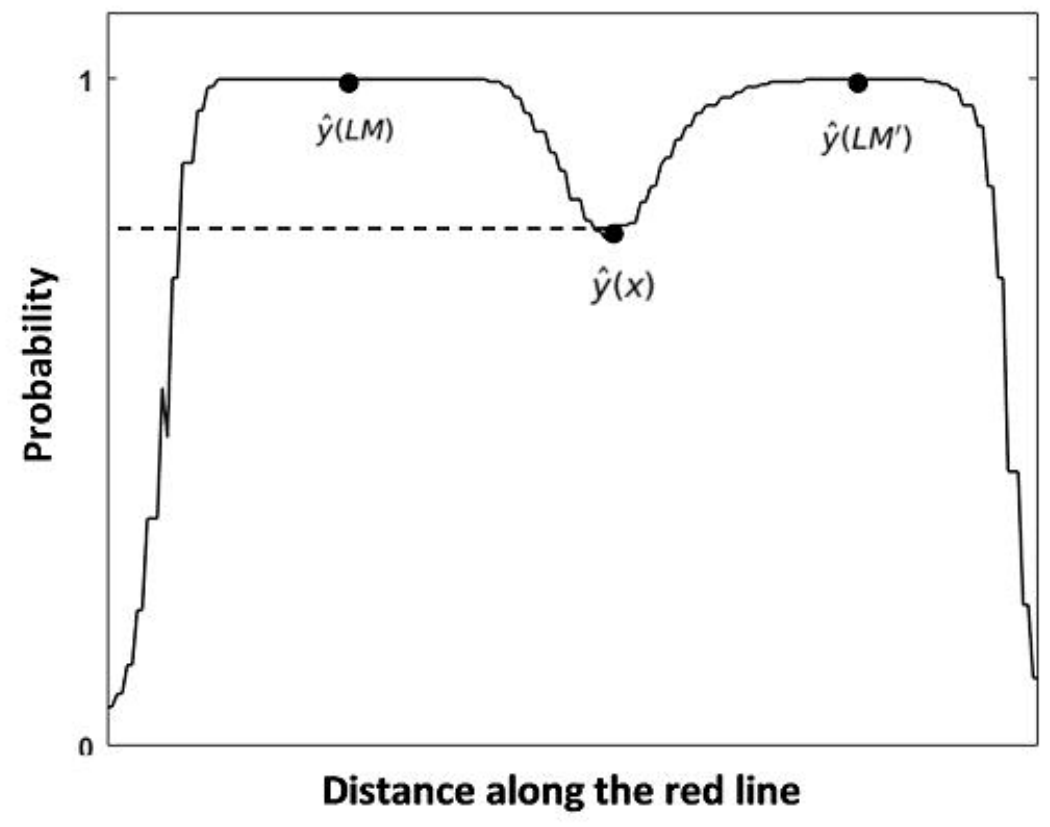


A.
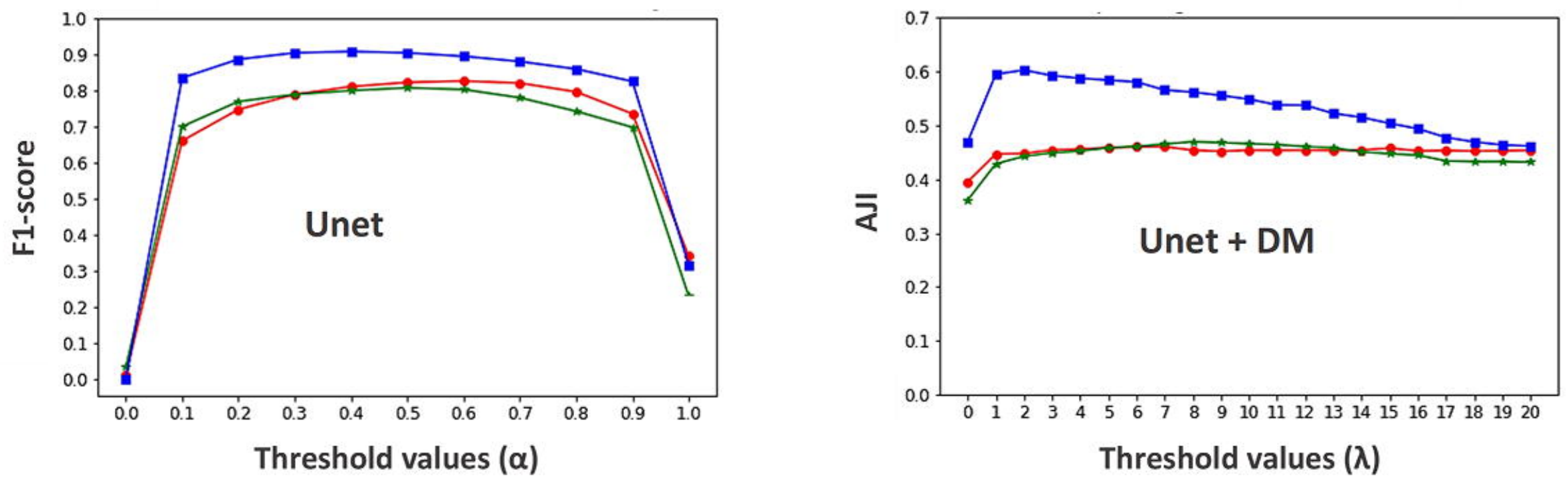

B.
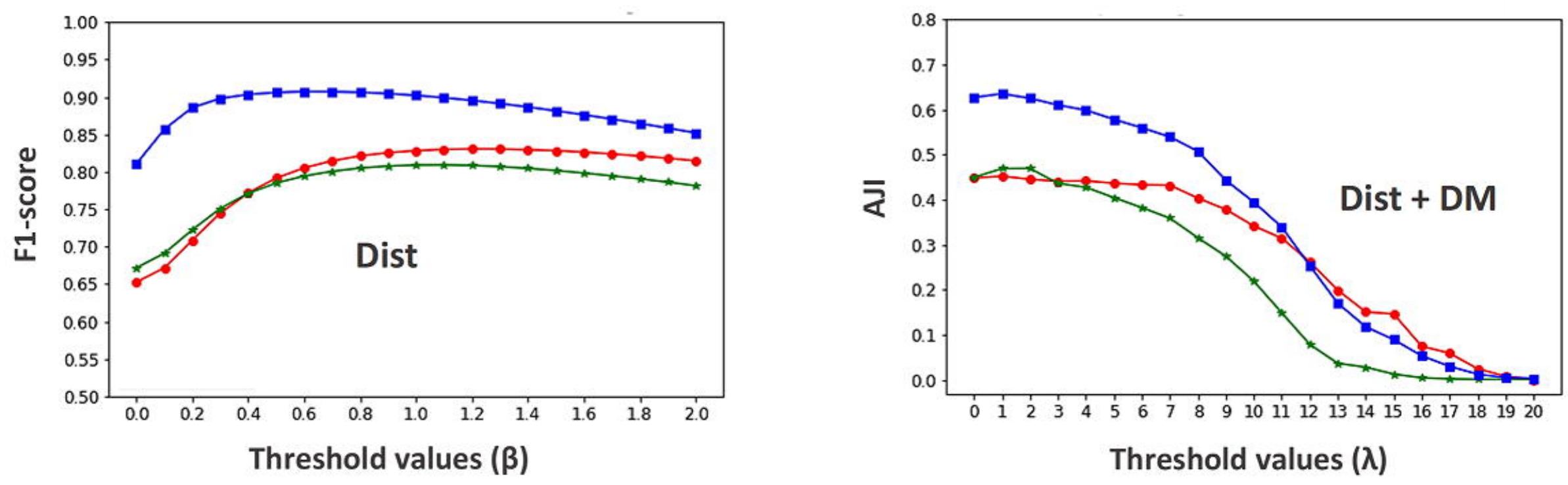

C.

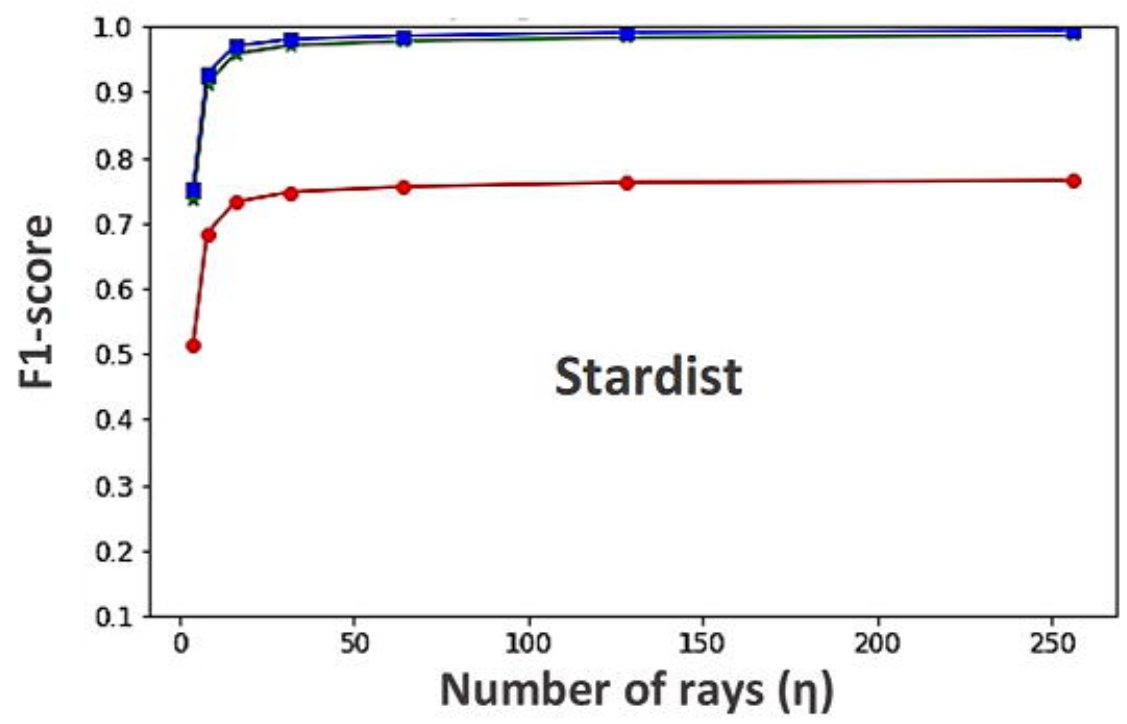

